\title{
Stochastic Inventory Management for Tactical Process Planning under Uncertainties: MINLP Models and Algorithms
}

\author{
Fengqi You, Ignacio E. Grossmann \\ Department of Chemical Engineering, Carnegie Mellon University \\ Pittsburgh, PA 15213, USA
}

December, 2009

\begin{abstract}
We address in this paper the mid-term planning of chemical complexes with integration of stochastic inventory management under supply and demand uncertainty. By using the guaranteed service approach to model the time delays in the chemical flows inside the chemical process network, we capture the stochastic nature of the supply and demand variations, and develop an equivalent deterministic optimization model to minimize the total cost including production cost, feedstock purchase cost, cycle inventory and safety stock costs. The model simultaneously determines the optimal purchases of the feedstocks, production levels of the processes, sales of final products and safety stock levels of all the chemicals, as well as the internal demand of the production processes. The model also captures "risk-pooling" effects to allow centralization of inventory management for chemicals that are consumed/produced by multiple processes. We formulate the model as a mixed-integer nonlinear program (MINLP) with a nonconvex objective function and nonconvex constraints. To solve the global optimization problem with modest computational times, we exploit some model properties and develop a tailored branch-and-refine algorithm based on successive piece-wise linear approximation. Five examples are presented to illustrate the application of the models and the performance of the proposed algorithm.
\end{abstract}

Key words: tactical planning, MINLP, stochastic inventory control, chemical process network 


\section{Introduction}

The chemical process industry often constructs large production sites, namely integrated chemical complexes ${ }^{1-3}$ that are composed of many interconnected processes and various chemicals. The integrated chemical complexes allow the chemical production to take advantage of synergies between processes. However, the risks associated with demand uncertainty and supply disruptions or delays may significantly affect tactical decision-making of a chemical complex. ${ }^{4-9}$ Although inventory improves the service by helping deal with demand uncertainty and providing flexibility, excessive inventory can be costly. ${ }^{10,11}$ In addition, a chemical complex usually involves many chemicals, including feedstocks, intermediates and final products, making it a non-trivial task to determine which chemicals should be stored and what is the optimal inventory level for each of them so as to achieve a certain service level and production target. Thus, cost-effective and agile inventory and production management can provide a competitive advantage for a company in a highly dynamic market. ${ }^{12,13}$ Therefore, it is of significant importance to integrate the tactical process planning decisions with the stochastic inventory management decisions across the entire chemical complex, and coordinate the activities of purchase, production, storage and sale to minimize the total cost. There are several challenges to achieve this goal.

The first challenge is how to model the inventory system of a chemical complex, which is more difficult than a multi-echelon inventory system and sometimes involves recycle flows. The second one is how to explicitly account for the supply delay and demand uncertainty in the inventory management and production planning. The third challenge is how to integrate the planning of purchase, production and sale, with inventory control, and how to model the information transformation to quantify the internal demand uncertainty of each processes. The last challenge is how to effectively solve the resulting optimization problem that leads to a large-scale nonconvex mixed-integer nonlinear program.

In this paper, we consider the medium term planning (typically 1- 6 months for process companies) of chemical process networks with integration of stochastic inventory management to deal with supply and demand uncertainty. By using the guaranteed service approach ${ }^{14-17}$ to model the time delays in the chemical flows, we capture the stochastic nature of the supply and demand uncertainty. An equivalent deterministic optimization model is developed to minimize the total cost including 
production cost, feedstock purchase cost, cycle inventory and safety stock costs. The model takes into account multiple tradeoffs and simultaneously determines the optimal purchase amount of each feedstock, production levels in each process, sale amount of each final product and inventory level of each chemical in the chemical process network, as well as the internal demand of the production processes. The model also captures the risk-pooling effect ${ }^{18}$ to allow centralization of safety stock management for chemicals that are consumed/produced in multiple processes. We first formulate the model as a mixed-integer nonlinear program (MINLP) with a nonconvex objective function and nonconvex constraints. To solve the problem with modest computational times, a tailored branch-and-refine algorithm based on successive piece-wise linear approximation is developed to for the global optimization. Five examples of chemical complexes with up to 38 processes and 28 chemicals are presented to illustrate the application of the model and the performance of the proposed algorithm.

The outline of this paper is as follows. We first review the related literature and the stochastic inventory modeling approach in the next section. The general problem statement is provided after the next section, which is followed by the model formulation for the problem of joint stochastic inventory management and production planning of a chemical process network. In the section of "Illustrative Examples", we present the results for three case studies of chemical complexes. To solve the large scale problem, a global optimization algorithm based on successive piecewise linear approximation is presented in the Section "Solution Algorithm". The computational results for large-scale instances and the conclusion of this paper are then given at the end of this paper.

\section{Literature Review}

The problem of planning under uncertainty for process network has been extensively studied in the past 20 years, but the inventory issue is usually neglected or coarsely considered without detailed inventory management policy. ${ }^{6-10,13,19-29}$ In these models, the safety stock level is given as a parameter, and is usually imbedded by including inventory lower bounds of various chemicals, or referred to as a "target inventory level" that would lead to some penalty costs if violated. This approach cannot optimize the safety stock levels, especially when considering supply and demand uncertainty. Thus, it can only provide an approximation of the inventory cost, and may 
lead to suboptimal solutions. Recently, Jung et al..$^{5}$ employ a simulation-optimization framework to determine the optimal safety stocks levels of a supply chain with consideration of production capacity.

Another body of research closely related to this work is the multi-echelon stochastic inventory theory, which can be traced back to the works by Simpson ${ }^{30}$ and by Clark and Scarf. ${ }^{31}$ There are two major approaches to model the multi-echelon inventory system: the stochastic service approach, and the guaranteed service approach. For a detailed comparison of these two approaches, see Graves and Willems, ${ }^{15}$ and Humair and Willems. ${ }^{32}$ Both approaches have pros and cons: stochastic service approach allows a more exact system understanding that can serve as the building block for more complex systems; guaranteed service approach models the entire system, which allows a planner to make tactical decisions without the need to approximate portions of the system that are not captured by a simplified topological representation. Based on this reason, we choose the guaranteed service approach to model the multi-echelon inventory system in this work since we focus on the modeling of inventory allocation across the entire process network. Although guaranteed service approach has been applied to address diverse problems in multi-echelon stochastic inventory management and supply chain optimization, ${ }^{14,}$ 16, 33-37 it has not been extended to the inventory systems of chemical complexes that include the production mode for arbitrary network topologies. Moreover, integrating stochastic inventory management into the tactical planning of process network is non-trivial, and has not been addressed in the existing literature to the best of our knowledge.

\section{Guaranteed Service Approach for Multi-Stage Stochastic Inventory Systems}

In this section, we briefly review some concepts of guaranteed service approach, which is the stochastic inventory management model used in this work. Detailed discussions about this approach are given by Graves and Willems ${ }^{15}$ and You and Grossmann. ${ }^{36}$

The main idea of the guaranteed service approach is that the service level for each node in the multi-echelon inventory system is fixed, and thus the optimal inventory level at each node is a function of the worst case replenishment lead time, which is a variable depending on the uncertain demand distribution of this node and the inventory 
level of its upstream node(s). For example, when Node 2 requests a replenishment from its upstream Node 1, the replenishment will arrive after a deterministic processing time $P_{2}$, if Node 1 has sufficient inventory to satisfy the demand from Node 2, as shown in Figure 1(a). However, if the inventory level in Node 1 is less than the internal demand from Node 1, Node 1 needs to further wait for the replenishment from its upstream predecessor so as to satisfy the demand from Node 2. In this case, it first takes some "waiting time" for the replenishments from the predecessor to arrive at Node 1, and then the deterministic processing time $P_{2}$ for the replenishment from Node 1 to arrive at Node 2. Thus, the replenishment lead time of Node 2 is equal to the "waiting time" plus the deterministic processing time. It is important to note that the "waiting time" is not deterministic, but similarly depends on the inventory levels of all the upstream nodes, and thus it is an uncertain variable. Since Node 2 has a fixed service level, the longer the worst case "waiting time" is, the more inventories should be hold in Node 2 to deal with the uncertain demand and lead time.

To quantify the "waiting time" for the worst case, the guaranteed service approach assumes that each node $j$ in the multi-echelon inventory system guarantees a service time $S_{j}$, which is the maximum time that all the demand from its downstream nodes (successor) will be satisfied. Besides, each node $j$ has a net lead time $N_{j}$, which is the required time span to cover demand variation with safety stocks at this node. Thus, The timing relationships between the deterministic process time $P_{j}$ and the times, including the guaranteed service time $S_{j}$, the worst case replenishment lead time $T_{j}$, and the net lead time $N_{j}$, are given as follows.

(a) The worst case replenishment lead time $T_{j}$ should be greater than or equal to the summation of the guaranteed service time of a direct predecessor $S_{j-1}$ and the processing time $P_{j}$ from the direct predecessor to this node, i.e. $T_{j} \geq S_{j-1}+P_{j}$.

(b) The net lead time $N_{j}$ equals to the difference between the worst case replenishment lead time $T_{j}$ and the guaranteed service time (GST) to its direct successor $S_{j}$, i.e. $N_{j}=T_{j}-S_{j}$.

These timing relationships are shown in Figure 1b. The relationship (a) follows directly from the aforementioned example. The relationship (b) is due to the reason that 
not all the customer demand of node $j$ at time $t$ must be satisfied immediately, but by the time $t+S_{j}$. Thus, the safety stocks of node $j$ do not need to cover demand variations over the entire worst case replenishment lead time, but just the difference between the replenishment lead time and the GST to the successors, i.e. the net lead time $N_{j}$. The relationships (a) and (b) imply that if the service time of node $j$ equals to the replenishment lead time, i.e. $T_{j}=S_{j}$ and $N_{j}=0$ as shown in Figure 1c, no safety stock is required in node $j$ because all the downstream demand only needs to be satisfied within the replenishment lead time, i.e. this node is operating in "pull" mode. If the guaranteed service time $S_{j}$ is 0 , i.e. $N_{j}=T_{j}$ as shown in Figure 1 d, the node holds the most safety stock because all the demand from the successors are satisfied immediately, i.e. this node is operating in "push" mode.

In the guaranteed service approach, each stage in the inventory system is assumed to operate under a base-stock policy, which is widely employed for inventory management across diverse sectors, with a common review period. ${ }^{5,38,39}$ Furthermore, demand over any time interval is assumed to be normally distributed, e.g. mean $\mu_{j}$ and standard deviation $\sigma_{j}$ for the daily demand of node $j$ (if the unit of $\mu_{j}$ is ton/day, the unit of $\sigma_{j}$ will be ton $/ \sqrt{ }$ day). Besides, for node $j$, there is an associated safety stock factor $\lambda_{j}$, which is given and corresponds to the standard normal deviate of the required service level, i.e. $\operatorname{Pr}\left(z \leq \lambda_{j}\right)=\alpha$ where $\alpha$ is the service level and $z$ is a standard normally distributed random variable with $z \sim N(0,1)$. Since the demand rate follows normal distributioin, the uncertain demand over the net lead time also follows normal distribution $N\left(N_{j} \cdot \mu_{j}, N_{j} \cdot \sigma_{j}^{2}\right)$. This yields the safety stock of node $j$ as $S S_{j}=\lambda_{j} \sigma_{j} \sqrt{N_{j}}$, and the optimal base-stock level for the inventory position, including the inventory on-hand and inventory in-transit, of this node is given by $B S_{j}=N_{j} \mu_{j}+\lambda_{j} \sigma_{j} \sqrt{N_{j}}$, which equals to the expected demand over the net lead time plus the safety stock. The base-stock level represents the inventory upper bound, and the lower bound is given by the safety stock level if there is no demand uncertainty. Since the physical inventory level is expected to vary between its upper and lower bound if there is no uncertainty (safety stocks are used to hedge uncertain demand), the average on-hand inventory level is given by the safety stock level plus half of the 
expected demand over the net lead time, i.e. $\operatorname{In} v_{j}=N_{j} \mu_{j} / 2+\lambda_{j} \sigma_{j} \sqrt{N_{j}}$, where the first term $N_{j} \mu_{j} / 2$ is the average working inventory (or cycle stock). Note that we neglect pipeline inventory (work-in-process) in the total inventory cost, because the focus of this work is to model the physical storage levels and allocation of chemicals of a chemical complex instead of a supply chain. In addition, the review period has been taken into account as part of the order processing time and considered in the net lead time.

In the guaranteed service approach, the service times from the external suppliers to the inventory system and the service times to the external customers are exogenous inputs, which can be treated as parameters, in addition to the deterministic processing time $P_{j}$ and the safety stock factor $\lambda_{j}$. Most of the "virtual" times, including the guaranteed service time $S_{j}$ of internal nodes, the worst case replenishment lead time $T_{j}$, and the net lead time $N_{j}$, are variables to be optimized.

\section{Problem Statement}

We are given a process network (Figure 2) consisting of a set of dedicated processes $i \in I$ and a set of chemicals $j \in J$. Each process $i \in I$ has a fixed production capacity $\mathrm{Cap}_{i}$, a unit production $\cos t \delta_{i}$ and a production delay (or production time) $P D_{i}$. Since we consider dedicated processes in this work, the production delays are deterministic parameters that are given. The chemicals could be feedstocks, intermediates or final products, and each of them can be purchased from the suppliers, produced in the chemical complex and sold to the markets. For each chemical $j$, there is a unit inventory holding cost $h_{j}$ and a safety stock factor $\lambda_{j}$, corresponding to the standard normal deviate of the required service level. In every production process $i$, we are also given a mass balance coefficient $\eta_{i j}$ for chemical $j$ that is consumed or produced by this process. In addition, the deterministic transfer times from process $i$ that produces chemicals $j$ to the storage tank of this chemical, $\gamma_{i j}$, and the deterministic transfer time of chemical $j$ from its storage tank to process $i$ that consumed this chemical, $\theta_{i j}$, are also given and assumed to be deterministic.

The process network also includes a set of suppliers $k \in K$ and a set of markets 
$l \in L$. For every chemical $j$, each supplier $k$ has a supply lower bound $a_{j k}^{L}$ and supply upper bound $a_{j k}^{U}$, as well as a unit price $\Gamma_{j k}$. The guaranteed service time $S I_{j k}$ of chemical $j$ supplied by supplier $k$ is also known. The GSTs of suppliers represent the supply uncertainty in terms of service time under the worst case. For every external market $l$, we are given a normally distributed demand with mean $\bar{\mu}_{j l}$ and standard deviation $\bar{\sigma}_{j l}$ for chemical $j$, and a required maximum guaranteed service time $S O_{j l}^{U}$ of each chemical $j$ demanded by this market.

The problem is to simultaneously determine the production level of each process, the working inventory and safety stock levels of each chemical, as well as the purchase amounts from external suppliers and sale amounts to external markets for each chemical in order to minimize the total purchase, production and inventory cost.

\section{Model Formulation}

The model will be formulated as an MINLP problem, which will predict the production levels, inventory levels, and purchase and sale amounts of a chemical complex. To explicitly account for the complex interactions between different states of chemicals (feedstocks, intermediate and final products) and production processes, we use a chemical process network representation, which is given in Figure 3 for the example problem shown in Figure 2. In Figure 3, the numbers inside the boxes indicate processes, and the letters inside the circles are for the chemicals. As a physical interpretation, one could consider these chemical nodes with red circles as the stocking points or storage tanks of chemicals (e.g. see state-task-network ${ }^{40}$ model for scheduling). This representation can greatly facilitate the analysis and modeling of the complex interactions between stochastic inventory management and the purchase, production and sale activities. An MINLP model built based on this network is presented in the following sections. A list of indices, sets, parameters and variables are given in the Appendix.

\section{Stochastic Lead Time Constraints}

There is a multi-echelon inventory system imbedded in a chemical process network: both chemical nodes and process nodes are stages or echelons of a multi-echelon

inventory systems, but safety stocks and working inventories (cycle stocks) are only 
allowed to be maintained in the chemical nodes, i.e. all the processes are operating as a "pull" system with zero net lead time and zero inventory. The inventory model used in this work is based on the guaranteed service approach, in which the service levels of all the stages are known but lead times are variables. Thus, the inventory model is based on the timing relationships for all the process nodes and chemical nodes in the chemical process network. Thus, we analyze and model the timing relationships, between the deterministic processing time, GSTs, worst case replenishment lead times and net lead times, for all the process nodes and chemical nodes in the chemical process network.

\section{Timing balance of process nodes}

The first timing constraint for the process nodes is to define the worst case replenishment lead time. Since the production node is treated as a stage in the inventory system with zero inventories, the deterministic processing time should be the time delay of material flow from the "gate" of its direct predecessors to the "gate" of this production node. Thus, the deterministic processing time includes the transfer time from the storage tank to this process and the production delay of this process. It implies that if chemical $j$ is a feedstock of process $i$, then the worst case replenishment lead time of process $i\left(T P_{i}\right)$ should be greater than or equal to the sum of the GST of chemical $j$ to this process $\left(S C_{i j}\right)$, the transfer time $\left(\theta_{i j}\right)$ from storage tank of chemical $j$ to process $i$ and the production delay of process $i\left(P D_{i}\right)$. This relationship leads to the following inequality.

$T P_{i} \geq S C_{i j}+\theta_{i j}+P D_{i}, \quad \forall j, i \in I(j)$

where $I(j)$ is the subset of processes that consume chemical $j$.

Since the production nodes do not hold safety stocks, their net lead times are zero and the GSTs to the successors equal to the worst case replenishment lead time. Thus, if chemical $j$ is a product of process $i$, the guaranteed service time $\left(S P_{i j}\right)$ of process $i$ to its downstream storage tank for chemical $j$ is equal to the worst case replenishment lead time of process $i$.

$S P_{i j}=T P_{i}, \forall j, i \in O(j)$

where $O(j)$ is the subset of processes that produce chemical $j$. 


\section{Timing balance of chemical nodes}

Using a similar analysis, we can derive the timing constraints for each chemical node. If chemical $j$ is a product of process $i$, the worst case replenishment lead time of chemical $j\left(T C_{j}\right)$ should be greater than the sum of the service time $\left(S P_{i j}\right)$ of its direct predecessors, i.e. process $i$, and the transfer time $\left(\gamma_{i j}\right)$ from process $i$ to storage tank of chemical $j$.

$$
T C_{j} \geq S P_{i j}+\gamma_{i j}, \forall j, i \in O(j)
$$

If chemical $j$ is also supplied by external supplier $k$ with non-zero flow rate, the worst case replenishment lead time of chemical $j$ should be also greater than the service time of this external supplier, $S I_{j k}$. This relationship can be modeled by the following constraint,

$$
T C_{j} \geq S I_{j k} \cdot X_{j k}, \forall j, k \in S U P(j)
$$

where $X_{j k}$ is a binary variable that is 1 if part or all of the chemical $j$ in the chemical process network is from supplier $k$, and $S U P(j)$ is the subset of suppliers that can provide chemical $j$.

If chemical $j$ is a feedstock of process $i$, the GST of chemical $j$ to its downstream process $i\left(S C_{i j}\right)$ should be greater than the difference between the worst case replenishment lead time of chemical $j$, and the net lead time $\left(N_{i j}\right)$ of chemical $j$ for the demand from process $i$.

$$
S C_{i j} \geq T C_{j}-N_{i j}, \forall j, i \in I(j)
$$

If chemical $j$ is sold to external market $l$, the guaranteed service time $\left(S O_{j l}\right)$ of chemical $j$ to this market should be greater than the worst case replenishment lead time of chemical $j$ minus the net lead time $\left(\bar{N}_{j l}\right)$ of chemical $j$ for the demand from market $l$,

$$
S O_{j l} \geq T C_{j}-\bar{N}_{j l}, \forall j, l \in M K T(j)
$$

where $\operatorname{MKT}(j)$ is the subset of markets that have positive demand of chemical $j$.

In addition, the GST of chemical $j$ to external market $l$ should not exceed its upper bound.

$$
S O_{j l} \leq S O_{j l}^{U}, \forall j, l \in M K T(j)
$$




\section{Tactical Planning Constraints}

Since we consider the mid-term planning of process networks, the following constraints are introduced to model the production, purchase and sale activities, and account for the mass balance relationships.

\section{Capacity constraints:}

The production capacity is measured in terms of the production amount of the main product in each process. Thus, it requires that the production amount $\left(W_{i j}\right)$ of main product $j$ in process $i$ does not exceed the capacity, $\operatorname{Cap}_{i}$.

$W_{i j} \leq \operatorname{Cap}_{i}, \forall i, j \in M_{i}$

where $M_{i}$ is the subset of chemicals that is the main product of process $i$.

$\underline{\text { Purchase and sale constraint: }}$

If supplier $k$ is selected to supply chemical $j$, the purchase amount $\left(P u_{j k}\right)$ should lie between their lower and upper bounds; if not, the purchase amount should be zero. This relationship can be modeled by the following constraint.

$a_{j k}^{L} \cdot X_{j k} \leq P u_{j k} \leq a_{j k}^{U} \cdot X_{j k}, \forall j, k \in S U P(j)$

Since the unexpected demand are hedged against by safety stocks, the sale amount $\left(S a_{j l}\right.$ ) of chemical $j$ to market $l$ only needs to satisfy the mean value of the demand, $\bar{\mu}_{j l}$, as in the deterministic process planning. It yields the following constraint.

$$
S a_{j l} \geq \bar{\mu}_{j l}, \forall j, l \in M K T(j)
$$

\section{Mass balance constraints:}

For chemical $j$, the total input, including the total external purchase amount and the total production amount, should be equal to the total output, including the total amount consumed by internal processes and the total amount sold to external markets. This mass balance relationship is given by the following constraint.

$$
\sum_{k \in S U P(j)} P u_{j k}+\sum_{i \in O(j)} W_{i j}=\sum_{i \in I(j)} W_{i j}+\sum_{l \in M K T(j)} S a_{j l}, \forall j
$$

where $P u_{j k}$ is the purchase amount of chemical $j$ from supplier $k$, and $S a_{j l}$ is the sale amount of chemical $j$ to market $l$. Note that inventory is not included in the mass 
balance constraint (11), because we only consider single time period in this work.

The consumption and production amount $\left(W_{i j}\right)$ of chemical $j$ in process $i$ is linearly related to the production amount $\left(W_{i j^{\prime}}\right)$ of the main product $j$ ' for process $i$ with the mass balance coefficient $\eta_{i j}$. This mass balance relationship is given by the following constraint.

$W_{i j}=\eta_{i j} \cdot W_{i j^{\prime}}, \forall i, j \in C_{i}, j^{\prime} \in M_{i}$

where $M_{i}$ is the set of main product of process $i$.

\section{Internal Demand Quantification}

In addition to the net lead time, the inventory level of chemical $j$ also depends on the distribution of the uncertain demand. Since the final demand of each chemical in each market is uncertain and follows a normal distribution, the input amount of each feedstock for each process, i.e. the internal demand, is also uncertain. The challenge is that we are only given the mean $\left(\bar{\mu}_{j l}\right)$ and standard deviation $\left(\bar{\sigma}_{j l}\right)$ of this chemical's demand in the market $l$, without knowing the detailed distribution of the "internal" demands.

\section{Probability Distribution of Internal Demands}

Since the final demand at each market follows a normal distribution, it follows that the internal demands are also normally distributed due to the linear mass balance constraint (11), to Cramér's theory (if the sum of some independent real-valued random variables is a normal random variable, then both all these random variables must be normally distributed as well), and to the perfect splitting property of the Gaussian distribution (the sum/difference of independent normal random variables also follows normal distribution with a mean as the sum/difference of the means of those random variables and a variance as the sum/difference of those variances). Since the mass balance constraints (11) require that the net input of each chemical should be equal to the net output, and the sale constraint (10) considers production targets as the mean values of market demands, the consumption amount of the feedstock $j$ in process $i\left(W_{i j}\right)$ corresponds to the mean value of the associated internal demand.

To determine the variance of an internal demand, let us first consider their variance-to-mean (VTM) ratios. The VTM ratio is unchanged when a normal 
distribution is randomly split into a few distributions, i.e. there is a linear relationship between the variance and mean. For process $i$, if the demands of its products increase, its consumption amounts of feedstocks should also increase. Thus, to ensure a continuous and stable production of the process, and to hedge against the uncertain demand, the VTM ratios for all internal demands of its feedstocks, denoted as $R P_{i}$, should be the same. Since we know that the mean value of the internal demand of chemical $j$ in process $i, i \in I(j)$ is $W_{i j}$, which is the consumption amount of feedstock $j$ in this process, the variance $\left(V_{i j}\right)$ of the internal demand of chemical $j$ in process $i$ is then given by the following constraint.

$V_{i j}=R P_{i} \cdot W_{i j}, \forall j, i \in I(j)$

\section{Uncertainty Propagation through the Chemical Process Network}

Because the information flow transfers from downstream to upstream, i.e. from the markets to the suppliers, we can use a "backward" derivation to determine the VTM ratios of the internal demands.

Let us first consider the input and output relationship of a chemical node as shown in Figure 4. The successors of chemical node $j$ include process $i, i \in I(j)$ which has normally distributed demand of chemical $j$ with variance $V_{i j}$ and VTM ratio $R P_{i}$, and market $l, l \in M K T(j)$, which also has normally distribution demand of chemical $j$ with variance $\bar{V}_{j l}$, i.e. $\bar{V}_{j l}=\bar{\sigma}_{j l}^{2}$, and VTM ratio $\bar{R}_{j l}$, i.e. $\bar{R}_{j l}=\bar{\sigma}_{j l}^{2} / \bar{\mu}_{j l}=\bar{V}_{j l} / \bar{\mu}_{j l}$. Thus, the total demand of chemical $j$ from the markets and downstream processes also follows a normal distribution with a mean given by $\left(\sum_{i \in I(j)} W_{i j}+\sum_{l \in M K T(j)} S a_{j l}\right)$ and a variance given by $\left(\sum_{i \in I(j)} V_{i j}+\sum_{l \in M K T(j)} \bar{R}_{j l} \cdot S a_{j l}\right)$. Hence, the VTM ratio of the demand of chemical $j$ is given by $\left(\sum_{i \in I(j)} V_{i j}+\sum_{l \in M K T(j)} \bar{R}_{j l} \cdot S a_{j l}\right) /\left(\sum_{i \in I(j)} W_{i j}+\sum_{l \in M K T(j)} S a_{j l}\right)$. If we denote $R C_{j}$ as the VTM ratio of the demand of product $j$ in process $i, i \in O(j)$, then the value of $R C_{j}$ is determined by modeling the uncertainty propagation from the downstream to upstream through the chemical node. There are two potential cases for uncertainty propagation, the ideal case and the worst case. 
In the ideal case, $R C_{j}$ equals to the VTM ratio of the total demand of chemical $j$ from markets and downstream processes, i.e. $\left(\sum_{i \in I(j)} V_{i j}+\sum_{l \in M K T(j)} \bar{R}_{j l} \cdot S a_{j l}\right) /\left(\sum_{i \in I(j)} W_{i j}+\sum_{l \in M K T(j)} S a_{j l}\right)$. One can draw an analogy of this "ideal" uncertainty propagation to the "pooling" problem ${ }^{41}$ with flow rate similar to the total demand rate and the concentration similar to the VTM ratio, although in this case we model the information flow transfers from downstream to upstream. Thus, the uncertainty propagation through a chemical node in the ideal case can be modeled with the following constraint.

$$
R C_{j} \cdot\left(\sum_{i \in I(j)} W_{i j}+\sum_{l \in M K T(j)} S a_{j l}\right)=\sum_{i \in I(j)} V_{i j}+\sum_{l \in M K T(j)} \bar{R}_{j l} \cdot S a_{j l}, \forall j
$$

where the right hand side of this constraint involves the product of two variables, i.e. bilinear terms.

With constraint (15) we can see that the uncertainty propagation under the ideal case maintains the conservation of variance. However, in most cases, the level of uncertainty amplifies as the information flow transfers from downstream to upstream, i.e. the "bullwhip effect". 42 Thus, we address this issue by considering another type of uncertainty propagation - the "worst" case propagation.

In the worst case, the "outbound" information stream from a chemical node to one of its direct predecessors (note that information transfers from downstream to upstream) should have a VTM ratio equal to the maximum VTM ratios of all the "inbound" information stream of this chemical node. In other words, the VTM ratio $\left(R C_{j}\right)$ of the demand of product $j$ of process $i, i \in O(j)$, equals to the maximum VTM ratio of chemical $j$ in the downstream processes $i, i \in I(j)$, and markets $l, l \in M K T(j)$, i.e. $R C_{j}=\max \left\{\max _{i \in I(j)}\left\{R P_{i}\right\}, \max _{l \in M K T(j)}\left\{\bar{R}_{j l}\right\}\right\}$. This relationship can be modeled by the following two constraints.

$$
\begin{aligned}
& R C_{j} \geq R P_{i}, \quad \forall j, i \in I(j) \\
& R C_{j} \geq \bar{R}_{j l}, \quad \forall j, \quad l \in M K T(j)
\end{aligned}
$$

The differences and similarities of these two approaches will be illustrated in Examples 1-3. In general, the worst case approach is relatively conservative and may lead to higher inventories and service levels than the ideal case approach. From the 
modeling perspective, constraint (15) includes non-convex and nonlinear bilinear terms on the left hand side, while constraint (16) and (17) are simply linear constraints. Although there is no clear rule how the uncertainty propagates through a chemical process network from the markets to the suppliers, the ideal case approach and the worst case approach represent two extreme scenarios. Since the inventory has to deal with unexpected situations and accounts for the worst case, the "worst" case uncertainty propagation might be a more suitable approach. A detailed comparison of these two approaches will be presented in Example 3.

Besides the above constraints, we also need to consider how the VTM ratios change as the information flow transfers through a process node. The successors of a process node $i$ are all chemical nodes, as shown in Figure 5, and all these chemical nodes are the products of this process. Since the process operates in the "pull" mode and all the products are produced simultaneously with a linear mass balance relationship as in constraint (12), the VTM ratio for the all demands of its feedstocks, $R P_{i}$, should be greater than or equal to the corresponding ratio of any of its products. Thus, we have the following constraint to model this relationship.

$R P_{i} \geq R C_{j}, \forall j, i \in O(j)$

\section{Non-negative Constraints}

All continuous variables must be nonnegative and the binary variables should be 0 or 1 .

$$
\begin{aligned}
& S C_{i j} \geq 0, N_{i j} \geq 0, V_{i j} \geq 0, \forall j, i \in I(j) \\
& S P_{i j} \geq 0, \forall j, i \in O(j) \\
& W_{i j} \geq 0, \forall j \in C_{i} \\
& R P_{i} \geq 0, T P_{i} \geq 0, \forall i \\
& R C_{j} \geq 0, T C_{j} \geq 0, \forall j \\
& P u_{j k} \geq 0, \forall j, k \in S U P(j) \\
& S a_{j l} \geq 0, \quad S O_{j l} \geq 0, \bar{N}_{j l} \geq 0, \forall j, l \in M K T(j) \\
& X_{j k} \in\{0,1\}, \forall j, k \in S U P(j)
\end{aligned}
$$




\section{Objective function}

The objective of this problem is to minimize the total cost, including feedstock purchase cost, production cost and inventory cost.

The purchase cost is given by the summation of products of the unit price of chemical and the total purchase amount, $\sum_{k} \sum_{j \in S U P(k)} \Gamma_{j k} \cdot P u_{j k}$, where $\Gamma_{j k}$ is the unit purchase price of chemical $j$ from supplier $k$.

The production cost is given by the summation of products of the unit processing cost and the production amount of main product, $\sum_{i} \sum_{j \in M_{i}} \delta_{i} \cdot W_{i j}$, where $\delta_{i}$ is the unit production cost of process $i$.

The inventory cost includes cycle stock cost and safety stock cost. Since the net lead time of chemical $j$ for the uncertain demand from downstream process $i$ and external market are $N_{j i}$ and $\bar{N}_{j}$, respectively, the uncertain internal and external demand of chemical $j$ follows normal distribution with mean $\left(\sum_{l \in M K T(j)} \bar{N}_{j l} \cdot \bar{\mu}_{j l}+\sum_{i \in I(j)} N_{i j} \cdot W_{i j}\right)$ and variance $\left(\sum_{l \in M K T(j)} \bar{N}_{j l} \cdot \bar{V}_{j l}+\sum_{i \in I(j)} N_{i j} \cdot V_{i j}\right)$. Thus, the optimal cycle stock of chemical $j$ is given by $\left(\sum_{l \in M K T(j)} \bar{N}_{j l} \cdot \bar{\mu}_{j l}+\sum_{i \in I(j)} N_{i j} \cdot W_{i j}\right) / 2$, and the safety stocks of chemical $j$ is given by $\lambda_{j} \sqrt{\sum_{l \in M K T(j)} \bar{N}_{j l} \cdot \bar{V}_{j l}+\sum_{i \in I(j)} N_{i j} \cdot V_{i j}}$, where $\lambda_{j}$ is the safety stock factor of chemical $j$. Note that this approach is consistent with the "risk-pooling" effect, ${ }^{18}$ where we centralize the inventory management of chemical $j$ and aggregate all the downstream demands into the chemical node. As will be shown in Example 2, this centralized approach will lead to potential reduction of total cost when compared with the decentralized inventory management, i.e. maintain the inventory in each individual process of a chemical complex.

With the aforementioned cost components, the objective function for minimizing the total cost is given in (20). 


$$
\begin{aligned}
\min & \sum_{i} \sum_{j \in M_{i}} \delta_{i} \cdot W_{i j} \\
+ & \sum_{k} \sum_{j \in S U P(k)} \Gamma_{j k} \cdot P u_{j k} \\
+ & \sum_{j} \frac{h_{j}}{2} \cdot\left(\sum_{l \in M K T(j)} \bar{N}_{j l} \cdot \bar{\mu}_{j l}+\sum_{i \in I(j)} N_{i j} \cdot W_{i j}\right) \\
+ & \sum_{j}\left(h_{j} \cdot \lambda_{j} \sqrt{\sum_{l \in M K T(j)} \bar{N}_{j l} \cdot \bar{V}_{j l}+\sum_{i \in I(j)} N_{i j} \cdot V_{i j}}\right)
\end{aligned}
$$

where $h_{j}$ is the unit inventory holding cost of chemical $j$.

\section{Non-convex MINLP Model}

The constraints and objective function discussed above yield two MINLP models, one is for the "ideal" case uncertainty propagation, denoted as model (P1), and the other is for the "worst" case uncertainty propagation, denoted as model (P2). The model for ideal case includes constraints (1)-(15), (18)-(19) and the objective function (20). The model for worst case includes constraints (1)-(14), (16)-(19) and the objective function (20). Both models are nonconvex, because constraints (14) and (15) include bilinear terms, and the objective function (20) has both square root terms and bilinear terms.

\section{Illustrative Example}

To illustrate the application of the MINLP models (P1) and (P2), we first consider three small-scale examples.

\section{Example 1: Timing Relationship and Mass Balance}

The first example is for a chemical process network with 3 chemicals, 3 processes, 2 suppliers and 1 market, taken from Sahinidis \& Grossmann. ${ }^{3}$ The chemical process network of this problem is given in Figure 6.

In this problem, the safety stock factor $\lambda_{j}$ for all the chemicals are the same and equal to 2.0537 , corresponding to a $98 \%$ service level, i.e. $\operatorname{Pr}(z \leq 2.0537)=98 \%$ for $z \sim N(0,1)$. The demand of Chemical $\mathrm{C}$ in the market follows a normal distribution with a mean of 100 ton/day and a standard deviation of 20 ton/ $/$ day. The maximum GST of Chemical $\mathrm{C}$ to the market is fixed to zero. The deterministic transfer times from 
process $i$ to storage tank of chemical $j\left(\gamma_{i j}\right)$ and from storage tank of chemical $j$ to process $i\left(\theta_{i j}\right)$ are neglected and set to zero. The remaining input data are given in Table 1.

Table 1 Input data of Example 1

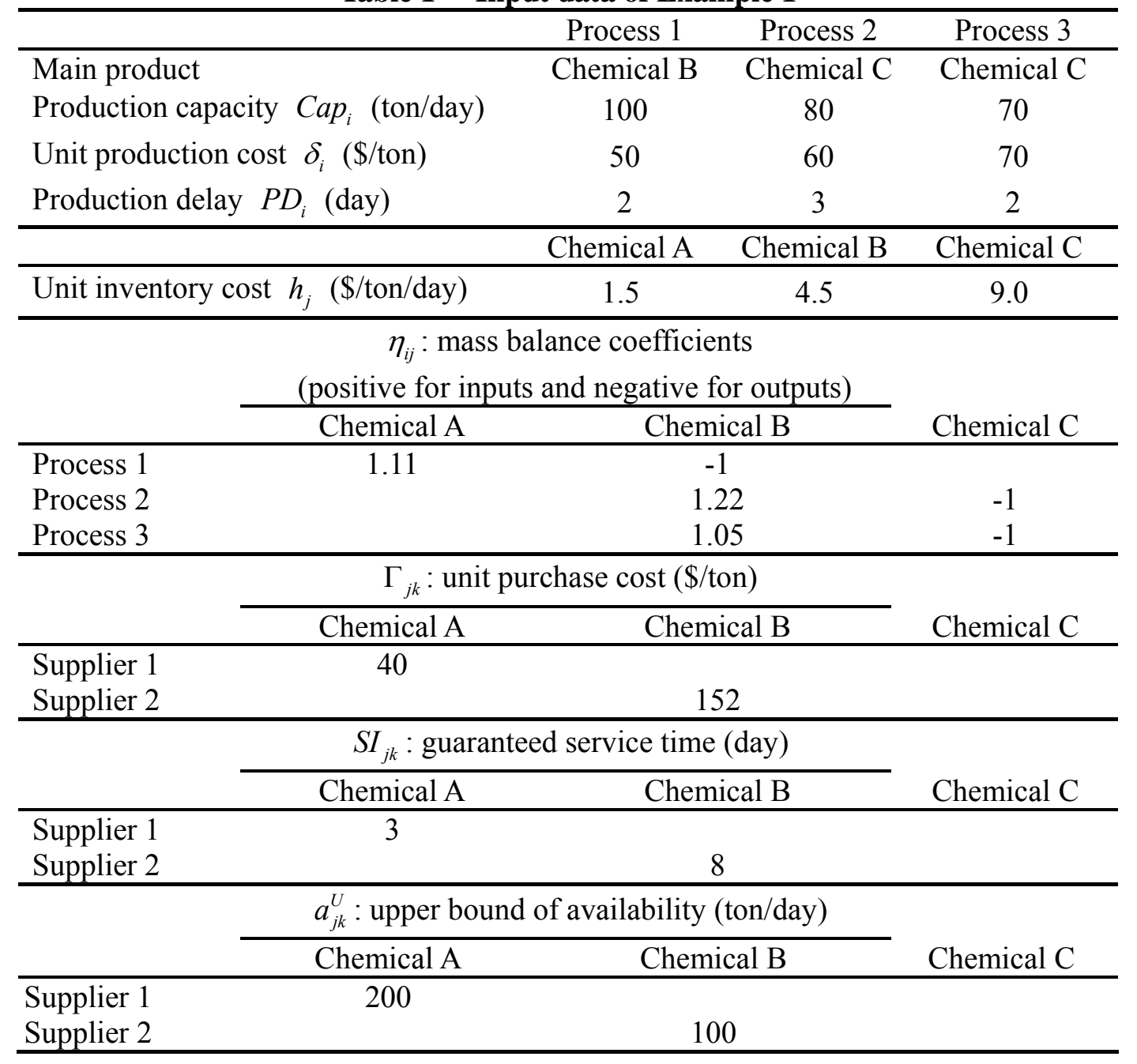

The MINLP model (P1) for "ideal case" uncertainty propagation involve 2 binary variables, 36 continuous variables and 38 constraints, and the MINLP model (P2) for "worst case" uncertainty propagation involve 2 binary variables, 36 continuous variables and 39 constraints. Since the problem sizes are rather small, we globally optimize both instances using the BARON solver ${ }^{43}$ with GAMS, ${ }^{44}$ and both CPU times are less than 1 second for obtaining optimal solutions with $0 \%$ optimality margin.

Both instances lead to the same optimal solution, and a minimum daily total cost of $\$ 22,007.07 /$ day. The reason is that this problem involves only one market, which only 
has demand of Chemical C. Thus, all the VTM ratios in this chemical process network equal to the VTM ratio of this final demand, no matter how we consider the uncertainty propagation. Note that in general cases, the "worst case" model (P2) may predict larger variances of internal demands, and consequently larger optimal safety stocks and higher total cost, compared to the "ideal case" model (P1).

The optimal solutions are shown in Table 2 and Figure 7 . We can see that the daily total safety stock cost of this chemical complex is $\$ 1,165.4$ day, which is around $5.3 \%$ of the daily total cost, and the total cost of cycle stocks is $\$ 3,166.4$ /day, which is around $14.4 \%$ of the daily total cost. The total purchase cost and total production cost represent $27.2 \%$ and $53.2 \%$ of the daily total cost, respectively. The production levels are given in Figure 7a. We can see that Process 1 and Process 3 are operating at full capacity. For Process 1 , the unit cost of Chemical B, including the unit production cost of Process 1 (\$50/ton) and the purchase cost of 1.11 tons of Chemical A from Supplier 1 (\$44.4/ton of Chemical B), is less than the purchase cost of Chemical from Supplier 2 (\$152/ton). For Process 3, although its unit production cost is higher than the one of Process 2, it requires less Chemical B as feedstock for the product of Chemical C, and consequently less unit cost of the final product. The purchase and sale activities are then coordinated based on the production level to ensure the mass balance in each process, supplier and market.

The detailed optimal net lead times and GSTs of all the nodes in this chemical process network are given in Figure $7 \mathrm{~b}$. The optimal solution shows that the chemical process network does not hold any inventory of the feedstock Chemical A, but the intermediate Chemical B and the final product Chemical C are both hold in storage. The reason is that the GST of supplier 1 plus the production delay of Process 1 is 5 days, but the GST of supplier 2 is 8 days. This implies that no matter how much inventory we hold for Chemical A, the worst case replenishment lead time for Chemical B is always 8 days. Thus, an economic way is to hold zero inventory of Chemical A, i.e. operating as "pull" system with zero net lead time, and quote a GST of 3 days to Process 1 . Now, consider the timing relationship between Chemical B and C and Process 2 and 3. Since the unit inventory cost of Chemical B (\$4.5/day/ton) is less the one for Chemical C (\$9/day/ton), it is optimal to hold as much inventory of Chemical B as possible, so as to reduce the required stock level of Chemical C. Thus, Chemical B quotes a GST of 0 day to Process 2 and allows its net lead time to Process 2 equal to its worst case replenishment lead time, 8 days. However, Chemical B guarantees a service time of 1 
day to Process 3, and has the corresponding net lead time as 7 days, instead of the maximum of 8 days. This is due to the difference between the production delays of Process 2 ( 3 days) and Process 3 ( 2 days). Since Chemical 3 needs to have zero GST to the market and holds inventories for a net lead time of 3 days, i.e. "push" system, it is optimal to have the production delay of Process 3 plus the GST to this process equal to 3 days as well. These results show that inventory allocation of the chemical process network leads to a hybrid "push - pull" system, where each node either does not hold inventory or holds maximum required inventory. What we observed in this example is consistent and similar to the phenomena discovered by Simpson $^{30}$ for multi-stage "series" inventory system. It is also interesting to note that all the optimal guaranteed service times and net lead times happen to be integer, although we do not restrict them to be integer values.

Table 2 Optimal solution of Example 1

\begin{tabular}{|c|c|c|c|c|}
\hline & & Chemical A & Chemical B & Chemical C \\
\hline \multicolumn{2}{|c|}{ Total safety stock (ton) } & 0 & 116.70 & 71.14 \\
\hline \multicolumn{2}{|c|}{ Total cycle stock (ton) } & 0 & 403.65 & 150 \\
\hline \multicolumn{2}{|c|}{ Total safety stock cost (\$/day) } & 0 & 525.3 & 640.3 \\
\hline \multicolumn{2}{|c|}{ Total cycle stock cost (\$/day) } & 0 & $1,816.4$ & 1,350 \\
\hline \multicolumn{5}{|c|}{$W_{i j}:$ production/consumption amount (ton/day) } \\
\hline & Chemical A & \multicolumn{2}{|c|}{ Chemical B } & Chemical C \\
\hline Process 1 & 111 & \multicolumn{2}{|c|}{100} & \\
\hline Process 2 & & \multicolumn{2}{|c|}{36.6} & 30 \\
\hline Process 3 & & \multicolumn{2}{|c|}{73.5} & 70 \\
\hline \multicolumn{5}{|c|}{$V_{i j}:$ variance of internal demand ( $\operatorname{ton}^{2} /$ day) } \\
\hline & Chemical A & \multicolumn{2}{|c|}{ Chemical B } & Chemical C \\
\hline Process 1 & 444 & & \\
\hline Process 2 & & \multirow{2}{*}{\multicolumn{2}{|c|}{$\begin{array}{c}146.4 \\
294\end{array}$}} & \\
\hline \multirow[t]{3}{*}{ Process 3} & & & & \\
\hline & \multicolumn{3}{|c|}{$P u_{j k}:$ purchase amount (ton/day) } & \\
\hline & Chemical A & \multicolumn{2}{|c|}{ Chemical B } & Chemical C \\
\hline Supplier 1 & 111 & \multirow{2}{*}{\multicolumn{2}{|c|}{10}} & \\
\hline \multirow[t]{3}{*}{ Supplier 2} & & & & \\
\hline & \multicolumn{3}{|c|}{$S a_{j l}:$ sale amount (ton/day) } & \\
\hline & Chemical A & & ical B & Chemical C \\
\hline \multicolumn{4}{|l|}{ Market } & 100 \\
\hline
\end{tabular}




\section{Example 2: Risk Pooling Effect}

The second example is for another chemical process network with 10 chemicals, 6 processes, 2 suppliers and 1 market, taken from Iyer \& Grossmann ${ }^{45}$ and shown in Figure 8a. In this problem, the safety stock factor for all the chemicals are also set to 2.0537 , corresponding to $98 \%$ service level. The maximum GST of all the chemicals to the market are zero, and the purchase lower bounds are all set to zero, too. The deterministic transfer times from process $i$ to storage tank of chemical $j\left(\gamma_{i j}\right)$ and from storage tank of chemical $j$ to process $i\left(\theta_{i j}\right)$ are neglected. All the external demands of chemicals in the market follow normal distributions. There are two suppliers, an international one (Supplier 1) and a domestic one (Supplier 2). In general, the international supplier has higher GSTs to supply the feedstocks, but lower unit purchase costs. The detailed input data are given in Table 3 .

Table 3 Input data of Example 2

\begin{tabular}{|c|c|c|c|}
\hline \multirow[b]{2}{*}{ Process 1} & \multicolumn{2}{|c|}{ production capacity $\mathrm{Cap}_{i}$ (ton/day) } & \multirow[b]{2}{*}{400} \\
\hline & 180 & Process 4 & \\
\hline Process 2 & 100 & Process 5 & 200 \\
\hline Process 3 & 50 & Process 6 & 100 \\
\hline \multicolumn{4}{|c|}{ unit production cost $\delta_{i}$ (\$/ton) } \\
\hline Process 1 & 55 & Process 4 & 50 \\
\hline Process 2 & 60 & Process 5 & 50 \\
\hline Process 3 & 70 & Process 6 & 90 \\
\hline \multicolumn{4}{|c|}{ production delay $P D_{i}$ (day) } \\
\hline Process 1 & 2 & Process 4 & 4 \\
\hline Process 2 & 3 & Process 5 & 5 \\
\hline Process 3 & 2 & Process 6 & 4 \\
\hline \multicolumn{4}{|c|}{ unit inventory cost $h_{j} \quad(\$ /$ ton/day $)$} \\
\hline Acetylene & 1.5 & Acrylonitrile & 8 \\
\hline Propylene & 2.5 & Isopropanol & 5 \\
\hline Benzene & 5.0 & Phenol & 3 \\
\hline Nitric Acid & 5.0 & Acetone & 7 \\
\hline Acetaldehyde & 10 & Cumene & 2 \\
\hline \multicolumn{4}{|c|}{ mass balance relationship } \\
\hline \multicolumn{4}{|c|}{0.63 Acetylene +0.58 Nitric Acid $=>$ Acrylonitrile } \\
\hline Process 2 & \multicolumn{3}{|c|}{0.64 Acetylene $=>0.55$ Nitric Acid + Acetaldehyde } \\
\hline Process 3 & \multicolumn{3}{|c|}{ 1.25 Propylene $=>0.9$ Nitric Acid + Acrylonitrile } \\
\hline Process 4 & \multicolumn{3}{|c|}{0.4 Propylene +0.69 Benzene $=>$ Cumene } \\
\hline Process 5 & \multicolumn{3}{|c|}{ 2.3 Cumene $=>1.7$ Phenol + Acetone } \\
\hline Process 6 & \multicolumn{3}{|c|}{0.74 Propylene $=>$ Isopropanol } \\
\hline
\end{tabular}




\begin{tabular}{|c|c|c|c|c|}
\hline \multicolumn{5}{|c|}{$\bar{\mu}_{j l}:$ mean value of external demands (ton/day) } \\
\hline Acetaldehyde & 80 & & Phenol & 200 \\
\hline Acrylonitrile & 110 & & Acetone & 100 \\
\hline Isopropanol & 70 & & Cumene & 70 \\
\hline \multicolumn{5}{|c|}{$\bar{\sigma}_{j l}:$ standard deviation of external demands (ton $/ \sqrt{ }$ day) } \\
\hline Acetaldehyde & 20 & & Phenol & 50 \\
\hline Acrylonitrile & 30 & & Acetone & 30 \\
\hline Isopropanol & 20 & & Cumene & 20 \\
\hline \multicolumn{5}{|c|}{$\Gamma_{j k}:$ unit purchase cost (\$/ton) } \\
\hline & Acetylene & Propylene & Benzene & Nitric Acid \\
\hline Supplier 1 & 550 & 400 & 440 & 820 \\
\hline Supplier 2 & 600 & 405 & 445 & 820 \\
\hline \multicolumn{5}{|c|}{$S I_{j k}:$ guaranteed service time (day) } \\
\hline & Acetylene & Propylene & Benzene & Nitric Acid \\
\hline Supplier 1 & 14 & 13 & 13 & 19 \\
\hline Supplier 2 & 2 & 3 & 2 & 4 \\
\hline \multicolumn{5}{|c|}{$a_{j k}^{U}:$ upper bound of availability (ton/day) } \\
\hline & Acetylene & Propylene & Benzene & Nitric Acid \\
\hline Supplier 1 & 100 & 200 & 200 & 50 \\
\hline Supplier 2 & 50 & 250 & 100 & 100 \\
\hline
\end{tabular}

In this example, we consider two instances, the centralized inventory management and the decentralized inventory management. The difference between them is that the centralized one takes into account the "risk pooling" effect ${ }^{18}$ in the inventory model, as in model (P1) and (P2), while the decentralized one does not group the inventories of the chemicals, but use a simple "rule of thumb" to maintain the individual storages for the feedstocks and/or products of all the processes and markets, i.e. each process manages its own inventories without coordination throughout the chemical complex. The chemical process networks of these two instances are given in Figure 8a and 8b, respectively, and the chemicals in these two chemical process networks are listed in Table 4. Compared to the centralized inventory management instance, the "decentralized" one has more chemical nodes due to the disaggregation of some chemicals as feedstocks and products of process.

Table 4 List of Chemicals in Example 2

\begin{tabular}{ll|cl}
\hline A & Acetylene & F & Acrylonitrile \\
B & Propylene & G & Isopropanol \\
C & Benzene & H & Phenol \\
D & Nitric Acid & I & Acetone \\
E & Acetaldehyde & J & Cumene \\
\hline
\end{tabular}


We first solved the instance for centralized inventory management with models (P1) and (P2) using GAMS/BARON, since there are only 8 binary variables, 108 continuous variables and 124 constraints in (P1) and 8 binary variables, 108 continuous variables and 128 constraints in (P2). The CPU times are both less than 1 second, and both models lead to the same global optimal solution with a minimum daily total cost of $\$ 312,288.81 /$ day. The reason is that all the VTM ratios of internal demands in the chemical process networks are the same in both optimal solutions, except those for the feedstocks of Process 4, Chemical B and C. However, the optimal net lead times of Chemical B to Process 4 and of Chemical $\mathrm{C}$ to Process 4 are zero in both optimal solutions. This implies that no safety stocks are held for the feedstocks of Process 4 . Therefore, the differences between the two approaches for uncertainty propagation are not reflected in the total minimum cost.

The optimal inventory levels and production, purchase and sale levels are shown in Table 5. The optimal total daily safety stock cost is $\$ 5,296.6 /$ day, which is around $1.7 \%$ of the daily total cost, and the total cost of cycle stocks is $\$ 13,390.4 /$ day, which is around $4.3 \%$ of the daily total cost. The total purchase cost and total production cost represent $81 \%$ and $13 \%$ of the daily total cost, respectively. Thus, the total inventory cost is almost half of the production cost, despite the high feedstock cost. In terms of the production levels, we can see from Table 5 that all the processes are operating with around $80 \%$ capacity due to the relatively low demand. We can also see that all the purchases of acetylene are from the internal supplier (Supplier 1) due to the relatively low price ( $\$ 550 /$ ton vs. $\$ 600 /$ ton). However, all the purchases of propylene are from the domestic supplier (Supplier 2), because the long lead time of the international supplier leads to higher inventory costs, which offsets its price advantage ( $\$ 400 /$ ton vs. $\$ 405 /$ ton). The results reveal the trade-offs between process planning and inventory management. It is interesting to note that due to the co-production of phenol and acetone in Process 5, the sale amount of acetone is higher than the production target.

Table 5 Optimal solution of the "centralized" inventory management Instance in Example 2

\begin{tabular}{lc|cc}
\hline \multicolumn{4}{c}{ production amount in terms of main product $W_{i j_{m}}$ (ton/day) } \\
\cline { 2 - 3 } Process 1 & 66.89 & Process 4 & 340.59 \\
Process 2 & 80 & Process 5 & 117.65 \\
Process 3 & 43.11 & Process 6 & 70 \\
\hline
\end{tabular}




\begin{tabular}{|c|c|c|c|}
\hline & \multicolumn{2}{|c|}{ total safety stock (ton) } & \multirow[b]{2}{*}{87.13} \\
\hline Acetylene & 188.35 & Acrylonitrile & \\
\hline Propylene & 96.56 & Isopropanol & 82.15 \\
\hline Benzene & 0 & Phenol & 229.61 \\
\hline Nitric Acid & 51.746 & Acetone & 137.77 \\
\hline Acetaldehyde & 71.142 & Cumene & 520.77 \\
\hline \multicolumn{4}{|c|}{ total cycle stock (ton) } \\
\hline Acetylene & 653.39 & Acrylonitrile & 110 \\
\hline Propylene & 158.53 & Isopropanol & 140 \\
\hline Benzene & 0 & Phenol & 500 \\
\hline Nitric Acid & 38.80 & Acetone & 250 \\
\hline Acetaldehyde & 120 & Cumene & 2,895 \\
\hline \multicolumn{4}{|c|}{$P u_{j k}:$ purchase amount (ton/day) } \\
\hline & Acetylene & Propylene & Benzene \\
\hline Supplier 1 & 93.34 & 0 & 200 \\
\hline Supplier 2 & 0 & 241.92 & 35.01 \\
\hline \multicolumn{4}{|c|}{$S a_{j l}:$ sale amount to the market (ton/day) } \\
\hline Acetaldehyde & 80 & Phenol & 200 \\
\hline Acrylonitrile & 110 & Acetone & 117.647 \\
\hline Isopropanol & 70 & Cumene & 70 \\
\hline
\end{tabular}

In the second instance of this problem we consider the decentralized inventory management, of which the chemical process network is given in Figure 8b. The models for the decentralized inventory management instance are almost the same as (P1) and (P2), except the safety stock cost term in the objective function. Recall that for chemical $j$, its uncertain demand from downstream process $i$ has a variance of $V_{i j}$ and a net lead time of $N_{j i}$, and its uncertain demand from downstream market $l$ (if applicable) has a variance of $\bar{V}_{j l}$ and a net lead time of $\bar{N}_{j}$. Thus, in the decentralized inventory management case, the optimal safety stocks of chemical $j$ for downstream process $i$ is $\lambda_{j} \sqrt{N_{i j} \cdot V_{i j}}$, and for market $l$ is $\lambda_{j} \sqrt{\bar{N}_{j l} \cdot \bar{V}_{j l}}$. Therefore, the total safety stock of chemical $j$ under the decentralized inventory management mode is $\lambda_{j}\left(\sum_{l \in M K T(j)} \sqrt{\bar{N}_{j l} \cdot \bar{V}_{j l}}+\sum_{i \in I(j)} \sqrt{N_{i j} \cdot V_{i j}}\right)$. Using a similar approach, it is easy to prove that the optimal cycle stocks of chemical $j$ is the same in both instances, and is given by $\left(\sum_{l \in M K T(j)} \bar{N}_{j l} \cdot \bar{\mu}_{j l}+\sum_{i \in I(j)} N_{i j} \cdot W_{i j}\right) / 2$. Therefore, the models for the decentralized inventory management have the same constraints as (P1) and (P2), but a new objective function is given in (21). 


$$
\begin{aligned}
\min & \sum_{i} \sum_{j \in M_{i}} \delta_{i} \cdot W_{i j} \\
+ & \sum_{k} \sum_{j \in S U P(k)} \Gamma_{j k} \cdot P u_{j k} \\
+ & \sum_{j} \frac{h_{j}}{2} \cdot\left(\sum_{l \in M K T(j)} \bar{N}_{j l} \cdot \bar{\mu}_{j l}+\sum_{i \in I(j)} N_{i j} \cdot W_{i j}\right) \\
+ & \sum_{j}\left[h_{j} \cdot \lambda_{j}\left(\sum_{l \in M K T(j)} \sqrt{\bar{N}_{j l} \cdot \bar{V}_{j l}}+\sum_{i \in I(j)} \sqrt{N_{i j} \cdot V_{i j}}\right)\right]
\end{aligned}
$$

We again use GAMS/BARON to solve the new models, which have the same variables and constraints as (P1) and (P2), respectively. Similarly to the previous instance, the ideal case and the worst case uncertainty propagation both lead to the same global minimum cost, $\$ 312,785.15 /$ day, of which $\$ 5,792.9$ day is safety stock cost, $\$ 13,390.4$ day is the cycle stock cost, $\$ 40,708.4$ day is the production cost and $\$ 252,893.4 /$ day is the purchase cost. The new models predict exactly the same optimal production, purchase and sales levels and costs as in the previous instance, but higher inventory levels. The optimal inventory levels of chemicals are listed in Table 6, and a comparison between the optimal safety stocks of the centralized and decentralized inventory management are presented in Figure 9, which shows that the safety stocks of acetylene, propylene and cumene have been significantly reduced by using centralized inventory management to account for "risk pooling", and in turn suggests the applicability of the proposed models.

Table 6 Optimal solution of the “decentralized” inventory management Instance in Example 2

\begin{tabular}{cc|cc}
\hline & \multicolumn{2}{c}{ total safety stock (ton) } & \\
\cline { 2 - 3 } Acetylene & 265.63 & Acrylonitrile & 87.13 \\
Propylene & 135.89 & Isopropanol & 82.15 \\
Benzene & 0 & Phenol & 229.61 \\
Nitric Acid & 51.75 & Acetone & 137.77 \\
Acetaldehyde & 71.14 & Cumene & 661.81 \\
\hline & \multicolumn{2}{c}{ total cycle stock (ton) } \\
Acetylene & 653.39 & Acrylonitrile & 110 \\
Propylene & 158.53 & Isopropanol & 140 \\
Benzene & 0 & Phenol & 500 \\
Nitric Acid & 38.80 & Acetone & 250 \\
Acetaldehyde & 120 & Cumene & 2,895 \\
\hline
\end{tabular}




\section{Example 3: Recycle and Uncertainty Propagation}

In the third example, we consider a chemical process network with 7 chemicals, 8 processes, 2 suppliers and 1 market. The chemical process network of this problem is given in Figure 10. In this example, we still consider 98\% service level for all the chemicals. The demands of Chemicals $\mathrm{D}$ and $\mathrm{G}$ in the market follow a normal distribution with mean values of 120 ton/day and 90ton/day, and standard deviation of 40 ton $/ \sqrt{ }$ day, 50 ton $/ \sqrt{ }$ day, respectively. The purchase lower bounds, the maximum GSTs of chemicals to the market, and the deterministic transfer times from process $i$ to storage tank of chemical $j\left(\gamma_{i j}\right)$ and from storage tank of chemical $j$ to process $i\left(\theta_{i j}\right)$ are all set to zero. The remaining input data are given in Table 7.

Table 7 Input data of Example 3

\begin{tabular}{|c|c|c|c|}
\hline & \multicolumn{2}{|c|}{ production capacity $\mathrm{Cap}_{i}$ (ton/day) } & \multirow[b]{2}{*}{50} \\
\hline Process 1 & 60 & Process 5 & \\
\hline Process 2 & 50 & Process 6 & 80 \\
\hline Process 3 & 90 & Process 7 & 80 \\
\hline Process 4 & 90 & Process 8 & 100 \\
\hline & \multicolumn{2}{|c|}{ unit production cost $\delta_{i}$ (\$/ton) } & \\
\hline Process 1 & 50 & Process 5 & 50 \\
\hline Process 2 & 60 & Process 6 & 90 \\
\hline Process 3 & 140 & Process 7 & 75 \\
\hline Process 4 & 20 & Process 8 & 100 \\
\hline & \multicolumn{2}{|c|}{ production delay $P D_{i}$ (day) } & \\
\hline Process 1 & 5 & Process 5 & 5 \\
\hline Process 2 & 3 & Process 6 & 4 \\
\hline Process 3 & 7 & Process 7 & 2 \\
\hline Process 4 & 4 & Process 8 & 4 \\
\hline & \multicolumn{2}{|c|}{ unit inventory cost $h_{j}(\$ /$ ton/day $)$} & \\
\hline A & 1.5 & $\mathrm{E}$ & 6 \\
\hline B & 5.5 & $\mathrm{~F}$ & 8 \\
\hline $\mathrm{C}$ & 7 & $\mathrm{G}$ & 5 \\
\hline $\mathrm{D}$ & 5 & & \\
\hline \multicolumn{4}{|c|}{ mass balance relationship } \\
\hline Process 1 & \multicolumn{2}{|c|}{$0.63 \mathrm{~A}=>\mathrm{B}$} & \\
\hline Process 2 & \multicolumn{2}{|c|}{$0.58 \mathrm{~A}=>\mathrm{B}$} & \\
\hline Process 3 & \multicolumn{2}{|c|}{$1.25 \mathrm{~B}=>4 \mathrm{C}+\mathrm{E}+1.5 \mathrm{~F}$} & \\
\hline Process 4 & \multicolumn{2}{|c|}{$0.1 \mathrm{~B}+0.05 \mathrm{D}=>\mathrm{C}$} & \\
\hline Process 5 & \multicolumn{2}{|c|}{$0.8 \mathrm{~B}=>\mathrm{E}$} & \\
\hline Process 6 & \multicolumn{2}{|c|}{$2.04 \mathrm{C}=>\mathrm{D}$} & \\
\hline Process 7 & \multicolumn{2}{|l|}{$2.3 \mathrm{C}=>\mathrm{D}$} & \\
\hline Process 8 & \multicolumn{2}{|c|}{$0.93 \mathrm{E}+0.77 \mathrm{~F}=>\mathrm{G}$} & \\
\hline
\end{tabular}




\begin{tabular}{lcc}
\hline & $\Gamma_{j k}:$ unit purchase cost (\$/ton) & \\
\cline { 2 - 3 } & $\mathrm{A}$ & $\mathrm{E}$ \\
\hline $\begin{array}{l}\text { Supplier 1 (international) } \\
\text { Supplier 2 (domestic) }\end{array}$ & 550 & 567 \\
& 820 & 840 \\
\hline & $S I_{j k}:$ guaranteed service time (day) & \\
\cline { 2 - 3 } & $\mathrm{A}$ & $\mathrm{E}$ \\
\hline Supplier 1 (international) & 12 & 2 \\
Supplier 2 (domestic) & 13 & 7 \\
\hline \multicolumn{2}{l}{$a_{j k}^{U}:$ upper bound of availability (ton/day) } \\
\cline { 2 - 3 } & $\mathrm{A}$ & $\mathrm{E}$ \\
\hline Supplier 1 (international) & 60 & 50 \\
Supplier 2 (domestic) & 100 & 100 \\
\hline
\end{tabular}

Table 8 Optimal solution of the "centralized" inventory management Instance in Example 2

\begin{tabular}{|c|c|c|c|}
\hline \multicolumn{4}{|c|}{ production amount in terms of main product $W_{i j_{m}}$ (ton/day) } \\
\hline Process 1 & 45.71 & Process 5 & 37.50 \\
\hline Process 2 & 50 & Process 6 & 80 \\
\hline Process 3 & 46.20 & Process 7 & 43.98 \\
\hline Process 4 & 79.55 & Process 8 & 90 \\
\hline \multicolumn{4}{|c|}{$P u_{j k}:$ purchase amount (ton/day) } \\
\hline & \multicolumn{2}{|c|}{ A } & $\mathrm{E}$ \\
\hline Supplier 1 (international) & \multicolumn{2}{|c|}{57.79} & $\overline{0}$ \\
\hline Supplier 2 (domestic) & \multicolumn{2}{|c|}{0} & 0 \\
\hline \multicolumn{4}{|c|}{$S a_{j l}:$ sale amount to the market (ton/day) } \\
\hline & \multicolumn{2}{|c|}{$\mathrm{D}$} & $\mathrm{G}$ \\
\hline Market & \multicolumn{2}{|c|}{120} & 90 \\
\hline & \multicolumn{2}{|c|}{ optimal cycle stock (ton) } & \\
\hline A & 317.76 & $\mathrm{E}$ & 0 \\
\hline $\mathrm{B}$ & 197.33 & $\mathrm{~F}$ & 0 \\
\hline $\mathrm{C}$ & 0 & G & 495 \\
\hline $\mathrm{D}$ & 675.91 & & \\
\hline \multicolumn{4}{|c|}{ optimal safety stock from Model (P1) (ton) } \\
\hline A & 266.91 & $\mathrm{E}$ & 0 \\
\hline B & 212.76 & $\mathrm{~F}$ & 0 \\
\hline $\mathrm{C}$ & 0 & $\mathrm{G}$ & 340.57 \\
\hline $\mathrm{D}$ & 275.72 & & \\
\hline \multicolumn{4}{|c|}{ optimal safety stock from Model (P2) (ton) } \\
\hline A & 272.87 & $\mathrm{E}$ & 0 \\
\hline B & 212.76 & $\mathrm{~F}$ & 0 \\
\hline $\mathrm{C}$ & 0 & $\mathrm{G}$ & 340.57 \\
\hline $\mathrm{D}$ & 275.72 & & \\
\hline
\end{tabular}


From Figure 10, we can see that the chemical process network of this example is different from the previous ones - there is a recycle from Process 6 and 7 to Process 4 . Recycle usually occur in chemical complexes, which makes it a challenging problem to employ a stochastic inventory approach to determine the optimal stock levels of this type of inventory systems. ${ }^{46}$ With the proposed model that integrates process planning with inventory management, we can explicitly address this challenge and handle it with the direct optimization approach without the need of detailed flow analysis. We solve the problem with the nonconvex MINLP models (P1) and (P2) using GAMS/BARON with $0 \%$ optimality margin. In contrast to the previous examples, the optimal solutions of using the "ideal" case uncertainty propagation and the "worst" case uncertainty propagation are different for this example. Model (P1), which is for the "ideal" case uncertainty propagation, leads to a global minimum daily cost of $\$ 78,572.72$ day, including \$4,652/day for safety stock cost, \$7,416.5/day for cycle stock cost, $\$ 34,717.5 /$ day for production cost and $\$ 31,786.7 /$ day for purchase cost. Model (P2), which is for the "worst" case uncertainty propagation, leads to a global minimum daily cost of $\$ 78,581.70 /$ day, including $\$ 4,660.9 /$ day for safety stock cost, $\$ 7,416.5 /$ day for cycle stock cost, $\$ 34,717.5 /$ day for production cost and $\$ 31,786.7 /$ day for purchase cost. Note that in this case the difference between the "ideal" and "worst" case is very small.

The optimal production, purchase, sale and inventory levels predicted by the two models are listed in Table 8 and Figure $11 \mathrm{a}-\mathrm{b}$. We can see that the optimal production, purchase and sale levels, as well as the cycle stock levels are the same for both solutions. In particular, Process 2 and Process 6 are operating with full capacity, while their parallel processes are not. The reason is that Process 2 has less unit production cost than Process 1, and Process 6 requires less feedstock than Process 7. In terms of inventory, it is interesting to note that the optimal solutions do not to hold inventories for intermediates - Chemical $\mathrm{C}, \mathrm{E}$ and $\mathrm{F}$, in order to achieve the optimal inventory allocation. In addition, we can see from Figure $11 \mathrm{~b}$ that the "worst" case approach leads to slightly higher safety stocks for Chemical A (272.87 tons vs. 266.91 tons), although the optimal cycle stocks are the same for both approaches. A comparison of the optimal VTM ratios with these two approaches is given in Figures 12. We can see that the ratios increase from downstream to upstream in both cases, and the ones for "ideal" case uncertainty propagation are always less than or equal to the ones for the "worst" case. Besides, the ratios are the same in both cases for the downstream chemicals and 
processes, but different for Chemical A and B and Process 1 and 2. The reason is that the information flows of demand uncertainty from Chemical D and "cross" with the one from Chemical $\mathrm{G}$ at the chemical node B. Due to the two different ways of quantifying the upstream demand uncertainties, the optimal VTM ratios for Chemical B are different in the two cases, and the difference consequently affects the upstream chemical nodes and process nodes.

From this example, we can also see that there are not too many differences between the optimal solutions predicted by the "ideal" and "worst" case models. Since model (P2) involves fewer nonconvex terms, and represents the most conservative "worst" case, it can be used to deal with the large-scale problem for higher computational efficiency without sacrificing accuracy. In addition, the main purpose of holding inventory is to deal with the unexpected situation and account for the worst case. To this end the "worst" case uncertainty propagation might be a more practical approach. In the next section, we first explore some properties of model (P2), and then the present an efficient computational algorithm, followed by another two large-scale examples.

\section{Solution Algorithm}

Although small scale problems can be solved to global optimality effectively by using a global optimizer, medium and large-scale problems are often computationally intractable with a direct solution approach due to the combinatorial nature and nonlinear nonconvex terms. In this section, we present an effective solution algorithm based on the model properties and successive piecewise linear approximation to globally optimize (P2) with modest computational expense.

\section{Reformulation}

In model (P2) with the "worst" case uncertainty propagation, the only nonconvex constraint is (14), which involves the VTM ratio and the internal demand's variance. Although the linear constraints (16)-(18) can be used to define the VTM ratios under the worst case, we can get rid of these constraints and determine the ratios in a pre-optimization step due to the following model property.

Property 1. At the optimal solution of problem (P2), the optimal variance-to-mean 
ratios of internal demands, denoted as $\left(R C_{j}^{*}, R P_{i}^{*}\right)$ must be equal to one of the variance-to-mean ratios of the final demands $\bar{R}_{j l}$. In other words, at the optimum $\exists j^{\prime}, l^{\prime}$ such that $R C_{j}^{*}=\bar{R}_{j^{\prime \prime l} l}$, and $\exists j ", l "$ such that $R P_{i}^{*}=\bar{R}_{j^{\prime \prime l} "}$.

Proof: If there is a positive directed flow from the chemical node $j$ to the chemical node $j$ ' which connects to market $l$ ', then due to the constraints (16)-(18), we can have $R C_{j} \geq \bar{R}_{j^{\prime} l}$. By figuring out all the possible flows that start from chemical node $j$ and end at another chemical node $j$ ', which has positive uncertain demand in the external market $l$ ', we can have $R C_{j} \geq \bar{R}_{j^{\prime} l^{\prime}}$ for $j$ and $j^{\prime}$ are on the same directed flow and $l^{\prime} \in M K T\left(j^{\prime}\right)$. Thus, the lower bound of $R C_{j}$ is given by $\max _{j^{\prime}, l^{\prime} \in M K T\left(j^{\prime}\right)}\left\{\bar{R}_{j^{\prime} l^{\prime}}\right\}$. Since the objective function is to minimize the total cost, including the safety stock cost, while a larger VTM ratio implies a higher variance of an internal demand, and consequently higher safety stock cost. Therefore, we can conclude that the optimal VTM ratio should lie at on its lower bound defined by the uncertainty propagation, i.e. $R C_{j}^{*}=\max _{j^{\prime}, l^{\prime} \in M K T\left(j^{\prime}\right)}\left\{\bar{R}_{j^{\prime} l^{\prime}}\right\}$. Using a similar approach, one can show that $R P_{i}^{*}=\max _{j^{\prime \prime}, l^{\prime \prime} \in M K T\left(j^{\prime \prime}\right)}\left\{\bar{R}_{j^{\prime \prime l} l}\right\}$ for $i$ and $j "$ are on the same directed flow and $l " \in M K T\left(j^{\prime \prime}\right)$.

Based on Property 1, we can determine the optimal VTM ratios in a pre-optimization step. Various path-search algorithms can be implemented to handle this issue. Alternatively, one can use the following algorithm that requires at most $\max \{|i|,|j|\}$ iterations.

Step1: Initialize iter $=1, R P_{i}^{\text {iter }}=0, R C_{j}^{\text {iter }}=0$. If $M K T(j) \neq \varnothing$, set $R C_{j}^{i t e r}=\max _{l \in M K T(j)}\left\{\bar{R}_{j l}\right\}$

Step2: For all $i, j$ such that $i \in O(j), \quad R P_{i}^{i t e r+1}=\max \left\{R P_{i}^{\text {iter }}, R C_{j}^{\text {iter }}\right\}$

Step3: For all $i, j$ such that $i \in I(j), R C_{j}^{\text {iter }+1}=\max \left\{R P_{i}^{i t e r}, R C_{j}^{i t e r}\right\}$

Step4: For all $j$ such that $M K T(j) \neq \varnothing, R C_{j}^{i t e r+1}=\max \left\{R C_{j}^{i t e r+1}, \max _{l \in M K T(j)}\left\{\bar{R}_{j l}\right\}\right\}$ 
Step5: Evaluate diff $=\sum_{j}\left(R C_{j}^{i t e r+1}-R C_{j}^{\text {iter }}\right)^{2}+\sum_{i}\left(R P_{i}^{i t e r+1}-R P_{i}^{\text {iter }}\right)^{2}$.

Step6: If diff $\leq 10^{-6}$, then set $R C_{j}^{*}=R C_{j}^{i t e r+1}$ and $R P_{i}^{*}=R P_{i}^{i t e r+1}$, and stop. Else, set iter $=$ iter +1 and go to Step 2.

For instance, applying this algorithm to Example 3, of which the chemical process network is given in Figure 10, requires 8 iterations. The values of $R C_{j}$ and $R P_{i}$ at each iteration are given in Table 9.

Table 9 Variance-to-mean ratios in each iteration

\begin{tabular}{ccccccccc}
\hline$R P_{i}$ & Iter. 1 & Iter. 2 & Iter. 3 & Iter. 4 & Iter. 5 & Iter. 6 & Iter. 7 & Iter. 8 \\
\hline Proc. 1 & 0 & 0 & 0 & 0 & 0 & 27.778 & 27.778 & 27.778 \\
Proc. 2 & 0 & 0 & 0 & 0 & 0 & 27.778 & 27.778 & 27.778 \\
Proc. 3 & 0 & 0 & 0 & 27.778 & 27.778 & 27.778 & 27.778 & 27.778 \\
Proc. 4 & 0 & 0 & 0 & 13.333 & 13.333 & 13.333 & 13.333 & 13.333 \\
Proc. 5 & 0 & 0 & 0 & 27.778 & 27.778 & 27.778 & 27.778 & 27.778 \\
Proc. 6 & 0 & 13.333 & 13.333 & 13.333 & 13.333 & 13.333 & 13.333 & 13.333 \\
Proc. 7 & 0 & 13.333 & 13.333 & 13.333 & 13.333 & 13.333 & 13.333 & 13.333 \\
Proc. 8 & 0 & 27.778 & 27.778 & 27.778 & 27.778 & 27.778 & 27.778 & 27.778 \\
\hline$R C_{j}$ & Iter. 1 & Iter. 2 & Iter. 3 & Iter. 4 & Iter. 5 & Iter. 6 & Iter. 7 & Iter. 8 \\
\hline $\mathrm{A}$ & 0 & 0 & 0 & 0 & 0 & 0 & 27.778 & 27.778 \\
$\mathrm{~B}$ & 0 & 0 & 0 & 0 & 27.778 & 27.778 & 27.778 & 27.778 \\
$\mathrm{C}$ & 0 & 0 & 13.333 & 13.333 & 13.333 & 13.333 & 13.333 & 13.333 \\
$\mathrm{D}$ & 13.333 & 13.333 & 13.333 & 13.333 & 13.333 & 13.333 & 13.333 & 13.333 \\
$\mathrm{E}$ & 0 & 0 & 27.778 & 27.778 & 27.778 & 27.778 & 27.778 & 27.778 \\
$\mathrm{~F}$ & 0 & 0 & 27.778 & 27.778 & 27.778 & 27.778 & 27.778 & 27.778 \\
$\mathrm{G}$ & 27.778 & 27.778 & 27.778 & 27.778 & 27.778 & 27.778 & 27.778 & 27.778 \\
\hline
\end{tabular}

With Property 1 we can determine the VTM ratios before solving the MINLP model (P2) and avoid the bilinear constraint (14). The second property of this model is about the timing relationship and is as follows.

Property 2. Denote $M$ as the maximum positive value such that for all the "timing" parameters $P D_{i}, S I_{j k}, S O_{j l}^{U}, \gamma_{i j}, \theta_{i j}$, have integer values for $P D_{i} / M, S I_{j k} / M$, $S O_{j l}^{U} / M, \gamma_{i j} / M, \theta_{i j} / M$, then at the optimal solution of (P2), all the optimal net lead times, denoted as $\left(N_{i j}^{*}, \bar{N}_{j l}^{*}\right)$ also have integer values for $N_{i j}^{*} / M$ and $\bar{N}_{j l}^{*} / M$.

Proof: Since $P D_{i} / M, S I_{j k} / M, S O_{j l}^{U} / M, \gamma_{i j} / M, \theta_{i j} / M$ are all integers, the 
stochastic lead time constraints (1)-(7) can be reformulated as follows:

$$
\begin{aligned}
& \frac{T P_{i}}{M}-\frac{S C_{i j}}{M} \geq \frac{\theta_{i j}}{M}+\frac{P D_{i}}{M}, \forall j, i \in I(j) \\
& \frac{S P_{i j}}{M}=\frac{T P_{i}}{M}, \forall j, i \in O(j) \\
& \frac{T C_{j}}{M}-\frac{S P_{i j}}{M} \geq \frac{\gamma_{i j}}{M}, \forall j, i \in O(j) \\
& \frac{T C_{j}}{M} \geq X_{j k} \cdot \frac{S I_{j k}}{M}, \forall j, k \in S U P(j) \\
& \frac{S C_{i j}}{M} \geq \frac{T C_{j}}{M}-\frac{N_{i j}}{M}, \forall j, i \in I(j) \\
& \frac{S O_{j l}}{M} \geq \frac{T C_{j}}{M}-\frac{\bar{N}_{j l}}{M}, \forall j, l \in M K T(j) \\
& \frac{S O_{j l}}{M} \leq \frac{S O_{j l}^{U}}{M}, \forall j, l \in M K T(j)
\end{aligned}
$$

Since the right hand side of constraints (1), (3), (4) and (7) are all integers, these stochastic lead time constraints define an integral polyhedron with all the extreme points $\left(N_{i j}^{*} / M, \bar{N}_{j l}^{*} / M, S O_{j l}^{*} / M, S C_{i j}^{*} / M, S P_{i j}^{*} / M, T C_{j}^{*} / M, T P_{i}^{*} / M\right)$ on the integer values. Note that these are the only constraints in model (P2) that relates to net lead time variables $N_{i j}$ and $\bar{N}_{j l}$. Besides, if we fix all the production, purchase and sale variables, the objective function is concave for variables $\left(N_{i j} / M\right)$ and $\left(\bar{N}_{j l} / M\right) .{ }^{35}$ Similarly to the problem addressed by You and Grossmann, ${ }^{35}$ this is a concave minimization problem over an integer polyhedron. Thus, the optimal solution always lies on the integer extreme points, ${ }^{47,48}$ i.e. $N_{i j}^{*} / M$ and $\bar{N}_{j l}^{*} / M$ must be integers.

Property 2 allows us to restrict $N_{i j} / M$ to be on integer values. Each integer variable $N_{i j} / M$ can be represented by a set of binary variables as follows: ${ }^{49}$

$$
\begin{aligned}
& N_{i j}=M \cdot \sum_{m} 2^{|m|-1} \cdot Z_{i j m}, \forall j, i \in I(j) \\
& Z_{i j m} \in\{0,1\}, \forall j, m, i \in I(j)
\end{aligned}
$$

where $Z_{i j m}$ determines the value of the $m$ th digit of the binary representation of 
$N_{i j} / M$. Note that the upper bound of $m$ depends on the upper bound of $N_{i j}$ and the value of $M$, which also depends on the timing parameters. For example, if $N_{i j}^{U}=50$ and $M=1$, we can set $m=1,2,3,4,5,6$.

With this transformation, we can reformulate model (P2) to reduce the nonlinearities. Substituting (22) into the objective function (20), it can then be reformulated as follows.

$$
\begin{aligned}
\min & \sum_{i} \sum_{j \in M_{i}} \delta_{i} \cdot W_{i j} \\
+ & \sum_{k} \sum_{j \in S U P(k)} \Gamma_{j k} \cdot P u_{j k} \\
+ & \sum_{j} \frac{h_{j}}{2} \cdot\left(\sum_{l \in M K T(j)} \bar{N}_{j l} \cdot \bar{\mu}_{j l}+M \cdot \sum_{i \in I(j)} \sum_{m} 2^{|m|-1} \cdot W_{i j} \cdot Z_{i j m}\right) \\
+ & \sum_{j}\left(h_{j} \cdot \lambda_{j} \sqrt{\sum_{l \in M K T(j)} \bar{N}_{j l} \cdot \bar{V}_{j l}+M \cdot \sum_{i \in I(j)} \sum_{m} 2^{|m|-1} \cdot Z_{i j m} \cdot V_{i j}}\right)
\end{aligned}
$$

Introducing two non-negative variables $G_{j}$ and $Q_{j}$, with the following equations:

$$
\begin{aligned}
& G_{j}=\sum_{l \in M K T(j)} \bar{N}_{j l} \cdot \bar{V}_{j l}+M \cdot \sum_{i \in I(j)} \sum_{m} 2^{|m|-1} \cdot Z_{i j m} \cdot V_{i j}, \forall j \\
& Q_{j}=\sum_{l \in M K T(j)} \bar{N}_{j l} \cdot \bar{\mu}_{j l}+M \cdot \sum_{i \in I(j)} \sum_{m} 2^{|m|-1} \cdot W_{i j} \cdot Z_{i j m}, \forall j
\end{aligned}
$$

Then the objective function can be further reformulated to (26) as follows.

$$
\min \sum_{i} \sum_{j \in M_{i}} \delta_{i} \cdot W_{i j}+\sum_{k} \sum_{j \in S U P(k)} \Gamma_{j k} \cdot P u_{j k}+\sum_{j} \frac{h_{j}}{2} \cdot Q_{j}+\sum_{j}\left(h_{j} \cdot \lambda_{j} \cdot \sqrt{G_{j}}\right)
$$

The linearization ${ }^{50}$ of $\left(Z_{i j m} \cdot V_{i j}\right)$ in constraint (24) requires two new continuous non-negative variable $Z V_{i j m}$ and $Z V 1_{i j m}$, and the following constraints,

$$
\begin{aligned}
& Z V_{i j m}+Z V 1_{i j m}=V_{i j}, \forall j, m, i \in I(j) \\
& Z V_{i j m} \leq V_{i j}^{U} \cdot Z_{i j m}, \quad \forall j, m, i \in I(j) \\
& Z V 1_{i j m} \leq V_{i j}^{U} \cdot\left(1-Z_{i j m}\right), \quad \forall j, m, i \in I(j) \\
& Z V_{i j m} \geq 0, Z V 1_{i j m} \geq 0, \forall j, m, i \in I(j)
\end{aligned}
$$

where constraints (27.2), (27.3), (27.4) ensure that if $Z_{i j m}$ is zero, $Z V_{i j m}$ should be zero; if $Z_{i j m}$ is one, $Z V 1_{i j m}$ should be zero. Combining with constraint (27.1), we can 
have $S Z_{j k}$ equivalent to the product of $S_{j}$ and $Z_{j k}$.

Similarly, the nonlinear terms $\left(W_{i j} \cdot Z_{i j m}\right)$ in constraint (25) can be linearized as follows,

$$
\begin{aligned}
& Z W_{i j m}+Z W 1_{i j m}=W_{i j}, \quad \forall j, m, i \in I(j) \\
& Z W_{i j m} \leq W_{i j}^{U} \cdot Z_{i j m}, \quad \forall j, m, i \in I(j) \\
& Z W 1_{i j m} \leq W_{i j}^{U} \cdot\left(1-Z_{i j m}\right), \forall j, m, i \in I(j) \\
& Z W_{i j m} \geq 0, Z W 1_{i j m} \geq 0, \quad \forall j, m, i \in I(j)
\end{aligned}
$$

where $Z W_{i j m}$ and $Z W 1_{i j m}$ are two new continuous variables, and $Z W_{i j m}$ is equivalent to $\left(W_{i j} \cdot Z_{i j m}\right)$.

Thus, constraints (24) and (25) can be replaced with the following equations.

$$
\begin{aligned}
& G_{j}=\sum_{l \in M K T(j)} \bar{N}_{j l} \cdot \bar{V}_{j l}+M \cdot \sum_{i \in I(j)} \sum_{m} 2^{|m|-1} \cdot Z V_{i j m}, \forall j \\
& Q_{j}=\sum_{l \in M K T(j)} \bar{N}_{j l} \cdot \bar{\mu}_{j l}+M \cdot \sum_{i \in I(j)} \sum_{m} 2^{|m|-1} \cdot Z W_{i j m}, \forall j
\end{aligned}
$$

With the above equations, we can reformulate model (P2) with a new model, denoted as (P3), which includes the objective function (26) and constraints (1)-(14), (19), (22), (27)-(30). Note that the value of $R P_{i}$ in constraint (14) is pre-determined, and thus all the constraints are linear in model (P3).

\section{Piece-wise Linear Approximation}

After the reformulation and linearization, we have the new model (P3) with all the constraints linear. The only nonlinear terms are in the objective function as univariate square root terms, $\sqrt{G_{j}}$. To improve the computational efficiency, we consider a piece-wise linear approximation for the concave square root terms. There are several different approaches to model piecewise linear functions for a concave term. In this work, we use the "multiple-choice" formulation ${ }^{51-54}$ to approximate the square root term $\sqrt{G_{j}}$. Let $P_{j}=\{1,2,3, \cdots, p\}$ denote the set of intervals in the piecewise linear function $\varphi\left(G_{j}\right)$, and $u_{j, 0}, u_{j, 1}, u_{j, 2}, \ldots u_{j, p}$, be the lower and upper bounds of $G_{j}$

for each interval. The "multiple choice" formulation of $\varphi\left(G_{j}\right)=\sqrt{G_{j}}$ is given by, 


$$
\varphi\left(G_{j}\right)=\min \sum_{p}\left(\beta_{j p} E_{j p}+\alpha_{j p} F_{j p}\right)
$$

s.t.

$\sum_{p} E_{j p}=1$

$\sum_{p} F_{j p}=G_{j}$

$u_{j, p-1} E_{j, p} \leq F_{j, p} \leq u_{j, p} E_{j, p}, \forall p$

$E_{j, p} \in\{0,1\}, F_{j p} \geq 0, \forall p$

where $\alpha_{j, p}=\frac{\sqrt{u_{j, p}}-\sqrt{u_{j, p-1}}}{u_{j, p}-u_{j, p-1}}$ and $\beta_{j, p}=\sqrt{u_{j, p}}-\alpha_{j, p} u_{j, p}, p \in P$.

Substituting (31) into the objective function (26) yields a mixed-integer linear programming (MILP) model (P4), which is piece-wise linear under-estimator of the MINLP model (P3). The model formulation of (P4) is given as follows.

$\min \sum_{i} \sum_{j \in M_{i}} \delta_{i} W_{i j}+\sum_{k} \sum_{j \in S U P(k)} \Gamma_{j k} P u_{j k}+\sum_{j} \frac{h_{j} Q_{j}}{2}+\sum_{j}\left(h_{j} \lambda_{j} \sum_{p}\left(\beta_{j p} E_{j p}+\alpha_{j p} F_{j p}\right)\right)$

s.t. Constraints (1)-(14), (19), (22), (27)-(30), (32)-(35)

As can be seen in Figure 13, the more intervals that are used in (P4), the better is the approximation of the nonlinear concave function $\sqrt{G_{j}}$, but more additional variables and constraints are required. Note that any feasible solution obtained from problem (P4) is also a feasible solution of (P3), and for each feasible solution the objective value of (P4) is always less than or equal to the objective value of (P3), i.e. the MILP model (P4) is a lower-bounding problem of the nonconvex MINLP problem (P3).

\section{Branch-and-Refine Algorithm}

In order to globally optimize the non-convex MINLP problem (P3), we can first solve the MILP problem (P4), whose solution provides a valid lower bound of the global optimal solution, and then solve a reduced nonlinear programming (NLP) problem by fixing the binary variables $X_{j k}$ and $Z_{i j m}$. Since the optimal solution of the reduced NLP is also a feasible solution of (P3), its objective value provides a valid global upper bound of the MINLP problem (P3). The remaining challenge is how to 
iteratively refine and improve the solution so that the global optimal solution can be obtained after a finite number of iterations. If we use a sufficiently large number of intervals in the piecewise linear approximation problem (P4), we are able to obtain the solution with sufficiently a small optimality margin. The reason is that the more intervals that are used, the better is the approximation of the square root function. However, more intervals require more additional variables and constraints in model (P3). To control the size of the problem, we use an iterative branch-and-refine strategy based on successive piece-wise linear approximation.

In the first step of this algorithm, we consider a one-piece linear approximation in (P4), i.e. replacing all the square root terms in (P3) with their secants as shown in Figure 14a. Thus, the optimal solution of the MILP problem (P4) provides the first lower bound LB1. Note that the optimal solution of (P4) is also a feasible solution of the MINLP problem (P3). Thus, an upper bound can be obtained by, a) substituting the optimal solution of (P3) into (P4) and directly evaluate the objective function, or b) fixing the values of the binary variables $X_{j k}$ and $Z_{i j m}$ and then solve the reduced NLP problem of (P4). Solving the reduced NLP may provide a better solution, but sometimes the NLP subproblems might become infeasible due to numerical difficulties. Thus, we use a combined approach: First, solve the reduced NLP; if it is feasible and return optimal solution, we move on to the next's step; if not, we use function evaluation with the optimal solution from (P4). The combined approach allows us to obtain an upper bound in each iteration, regardless if the reduced NLP is feasible or not.

In the next step, we use the optimal solution of variable $G_{j}$ in the upper bounding problem as the lower bound of a new interval, and consider a two-interval linear approximation of the square root terms as shown in Figure 14b. Note that for those $\sqrt{G_{j}}$, the optimal solution of the upper bounding problem in the previous iteration lies at the bounds of some intervals, then we do not add a new interval for these square root terms. After we construct the two-interval linear approximation MILP model (P4), we can similarly obtain a lower bound, and then an upper bound by function evaluation or solving the reduced NLP.

As shown in Figure 14c, as the iteration number increases, the number of intervals increases in (P4), and the best lower bound increases while the best upper bond decreases. The algorithm keeps iterating until the lower bound and upper bound are close enough to reach an optimality tolerance, e.g. $10^{-6}$. Note that the number of 
intervals does not always equal to the number of iterations, because the optimal solutions in some iterations may lie at the bounds of the intervals and in that case we do not increase a new interval for the corresponding square root terms.

To summarize, the proposed branch-and-refine algorithm based on successive piece-wise linear approximation is as follows:

\section{Step 1: (Initialization)}

Set iter $=1, L B=0, U B=+\infty$. Set $N P_{j}^{\text {iter }}=1, p \in P_{j}=\left\{0,1, \ldots, N P_{j}^{\text {iter }}\right\}$. Construct one-interval linear approximation, i.e. the secant, of the square root terms. To achieve this, we set $u_{j, 0}=0$ and $u_{j, 1}=G_{j}^{U}$, as well as $\alpha_{j, 1}=\frac{\sqrt{u_{j, 1}}-\sqrt{u_{j, 0}}}{u_{j, 1}-u_{j, 0}}=\frac{1}{\sqrt{G_{j}^{U}}}$ and $\beta_{j, 1}=\sqrt{u_{j, 1}}-\alpha_{j, 1} u_{j, 1}=0$

\section{Step 2:}

At iteration iter, solve the piece-wise linear approximation MILP model (P4). Denote the optimal objective function value as $\varphi^{\text {iter }}$ and the optimal solution of variables $X_{j k}$ and $Z_{i j m}$ as $\left(X_{j k}^{* i t e r}, Z_{i j m}^{* i t e r}\right)$. Fix the values of binary variables $X_{j k}=X_{j k}^{* i t e r}$ and $Z_{i j m}=Z_{i j m}^{* i t e r}$, and solve the original model (P3) in the reduced space as an NLP to obtain the local optimal objective function $\Phi^{\text {iter }}$ and optimal solution $G_{j}^{\text {iter }}$. If the reduced NLP problem (P3) is infeasible, then substitute the optimal solution of the MILP problem into the original model (which is always feasible) and evaluate the objective function value as $\Phi^{i t e r}$ and optimal solution $G_{j}^{\text {iter }}$.

\section{Step 3:}

If $\Phi^{\text {iter }}<U B$, then set $U B=\Phi^{\text {iter }}$, store the current optimal solution of the (P3). If the NLP is infeasible, store the optimal solution of the MILP model (P4).

If $\varphi^{\text {iter }}>L B$, then set $L B=\varphi^{\text {iter }}$.

Find $n_{j}^{\text {iter }}$ such that the optimal solution $G_{j}^{\text {iter }}$ lies in the $n_{j}^{\text {iter }}$ th interval. i.e. $u_{j, n_{j}^{\text {ter }}-1} \leq G_{j} \leq u_{j, n_{j}^{\text {iter }}}$. One approach to find the proper $n_{j}^{\text {iter }}$ is to compute the product 
$\left(G_{j}^{i t e r}-u_{j, p-1}\right) \cdot\left(G_{j}^{i t e r}-u_{j, p}\right)$ for all the $p=2,3, \ldots, N P_{j}^{i t e r}$, and then denote the first $p$ that leads to a non-positive value (zero or negative value) of the product as $n_{j}^{\text {iter }}$, i.e. if $\left(G_{j}^{i t e r}-u_{j, p-1}\right) \cdot\left(G_{j}^{i t e r}-u_{j, p}\right) \leq 0$, set $n_{j}^{i t e r}=p$.

If $U B-L B \leq \varepsilon$ (e.g. $\left.10^{-9}\right)$, stop and output the optimal solution; otherwise, go to the next step.

\section{Step 4:}

For those $j \in J$ such that $G_{j}^{\text {iter }}=u_{j, n_{j}^{i \text { ier }}}$, set $N P_{j}^{\text {iter }+1}=N P_{j}^{\text {iter }}, u_{j, p}^{\text {iter }+1}=u_{j, p}^{\text {iter }}$, $\alpha_{j, p}^{i t e r+1}=\alpha_{j, p}^{i t e r}$ and $\beta_{j, p}^{i t e r+1}=\beta_{j, p}^{i t e r}$.

For other $j \in J$, set $N P_{j}^{\text {iter }+1}=N P_{j}^{\text {iter }}+1$ and update set $P_{j}$, i.e. $p \in P_{j}=\left\{0,1, \ldots, N P_{j}^{\text {iter }}, N P_{j}^{i \text { ter }+1}\right\}$. Then update $u_{j, p}, \alpha_{j, p}$ and $\beta_{j, p}$ as follows:

(a) For $p<n_{j}^{\text {iter }}$ (i.e. $p=0,1,2 \ldots, n_{j}^{\text {iter }}-1$ ), set $u_{j, p}^{\text {iter }+1}=u_{j, p}^{\text {iter }}, \alpha_{j, p}^{\text {iter }+1}=\alpha_{j, p}^{\text {iter }}$ and $\beta_{j, p}^{i t e r+1}=\beta_{j, p}^{i t e r}$.

(b) For $p=n_{j}^{\text {iter }}, \quad$ set $\quad u_{j, n_{j}^{\text {iter }}}^{\text {iter } 1}=G_{j}^{\text {iter }}, \quad \alpha_{j, n_{j}^{\text {iter }}}^{\text {iter } 1}=\frac{\sqrt{G_{j}^{\text {iter }}}-\sqrt{u_{j, n_{j}^{\text {iter }}-1}}}{G_{j}^{\text {iter }}-u_{j, n_{j}^{\text {iter }}-1}} \quad$ and $\beta_{j, n_{j}^{\text {iter }}}^{\text {iter }+1}=\sqrt{G_{j}^{i t e r}}-\alpha_{j, n_{j}^{i t e r}}^{i \text { iter }} \cdot G_{j}^{i t e r}$

(c) For $p=n_{j}^{\text {iter }}+1, \quad$ set $\quad u_{j, n_{j}^{\text {iter }}+1}^{\text {iter+ }}=u_{j, n_{j}^{\text {iter }}}^{\text {iter }}, \quad \alpha_{j, n_{j}^{\text {iter }}+1}^{\text {iter }}=\frac{\sqrt{u_{j, n_{j}^{\text {iter }}}}-\sqrt{G_{j}^{\text {iter }}}}{u_{j, n_{j}^{\text {iter }}}-G_{j}^{\text {iter }}} \quad$ and $\beta_{j, n_{j}^{\text {iter }}+1}^{\text {iter }+1}=\sqrt{u_{j, n_{j}^{\text {iter }}}}-\alpha_{j, n_{j}^{\text {iter }}}^{\text {iter } 1} \cdot u_{j, n_{j}^{\text {iter }}}$

(d) For $p>n_{j}^{\text {iter }}+1$ (i.e. $\left.p=n_{j}^{\text {iter }}+2, n_{j}^{\text {iter }}+3, \ldots, N P_{j}^{\text {iter }}\right)$, , set $u_{j, p}^{\text {iter }+1}=u_{j, p-1}^{\text {iter }}$, $\alpha_{j, p}^{i t e r+1}=\alpha_{j, p-1}^{i t e r}$ and $\beta_{j, p}^{\text {iter }+1}=\beta_{j, p-1}^{\text {iter }}$.

Then, set iter $=$ iter +1 and go to Step 2.

We should note that the entire procedure requires only an MILP solver. An NLP solver can be used to solve the upper bounding problem in the reduced variable space in Step 2, but it is not necessary. The reason is that the solution of the nonlinear 
optimization problem (P3), which reduces to an NLP from MINLP after $X_{j k}$ and $Z_{i j m}$ are fixed, can be substituted by simple function evaluation as stated in Step 2, although the solution quality may be sacrificed.

\section{Computational Results}

In order to illustrate the performance of the proposed solution strategies, we consider two large-scale problems, Examples 4 and 5. All the computational experiments are performed on an IBM T400 laptop with Intel $2.53 \mathrm{GHz}$ CPU and $2 \mathrm{~GB}$ RAM. The proposed solution procedure is coded in GAMS 23.2.1. ${ }^{44}$ The MILP problems are solved using CPLEX 12, the NLP problems in Step 2 of the branch-and-refine algorithm are solved with solver KNITRO 6.0.0. We use DICOPT as the convex MINLP solver and the global optimizer used in the computational experiments is BARON 8.1.5. The optimality tolerances of DICOPT, BARON and the proposed algorithm are all set to $10^{-6}$ and optimality margins of solving the piecewise linear approximation MILP model (P4) and the reduced NLP model (P3) are both $10^{-9}$.

\section{Examples 4: Computational Performance, Inventory Allocation}

To test the performance of the proposed algorithm, we consider Example 4, which has 20 chemicals, 13 processes. The chemical process network of Example 4 is given in Figure 15. We consider 10 instances of this example, ranging from 1 supplier and 1 market instance to 10 supplier and 10 market instances.

Table 10 Input data of the first instance of Example 4 (1 supplier and 1 market)

\begin{tabular}{cc|cc}
\hline & \multicolumn{2}{c}{ production capacity $\operatorname{Cap}_{i}$ (ton/day) } & \\
\cline { 2 - 3 } Process 1 & 15 & Process 14 & 100 \\
Process 3 & 150 & Process 16 & 150 \\
Process 4 & 180 & Process 17 & 50 \\
Process 5 & 90 & Process 28 & 50 \\
Process 8 & 50 & Process 32 & 250 \\
Process 12 & 50 & Process 38 & 120 \\
Process 13 & 40 & \\
\hline & \multicolumn{2}{c}{ Prit production cost $\delta_{i}$ (\$/ton) } & 200 \\
Process 1 & 80 & Process 14 & 100 \\
Process 3 & 120 & Process 16 & 130 \\
Process 4 & 150 & Process 17 & 100 \\
Process 5 & 110 & Process 28
\end{tabular}




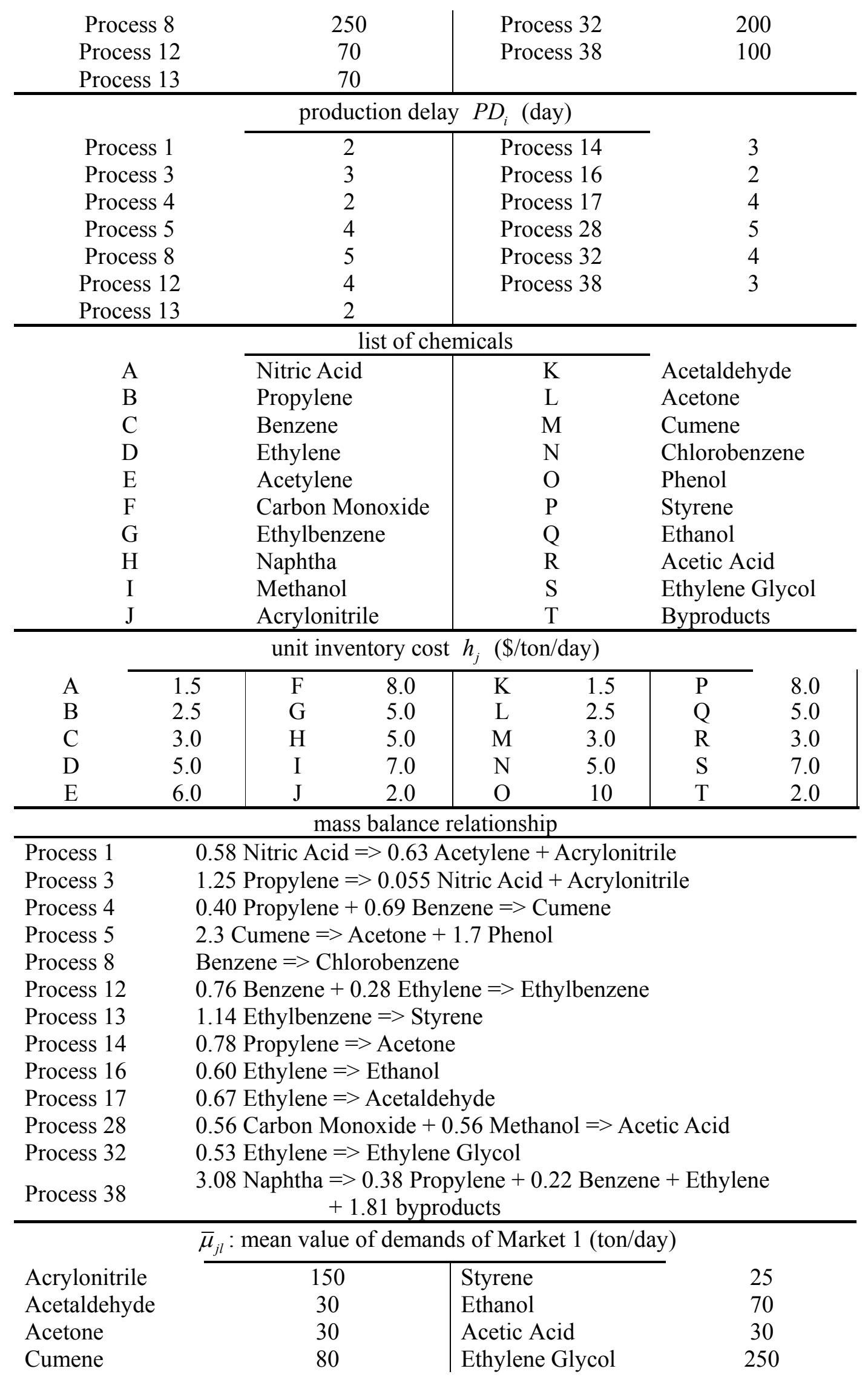




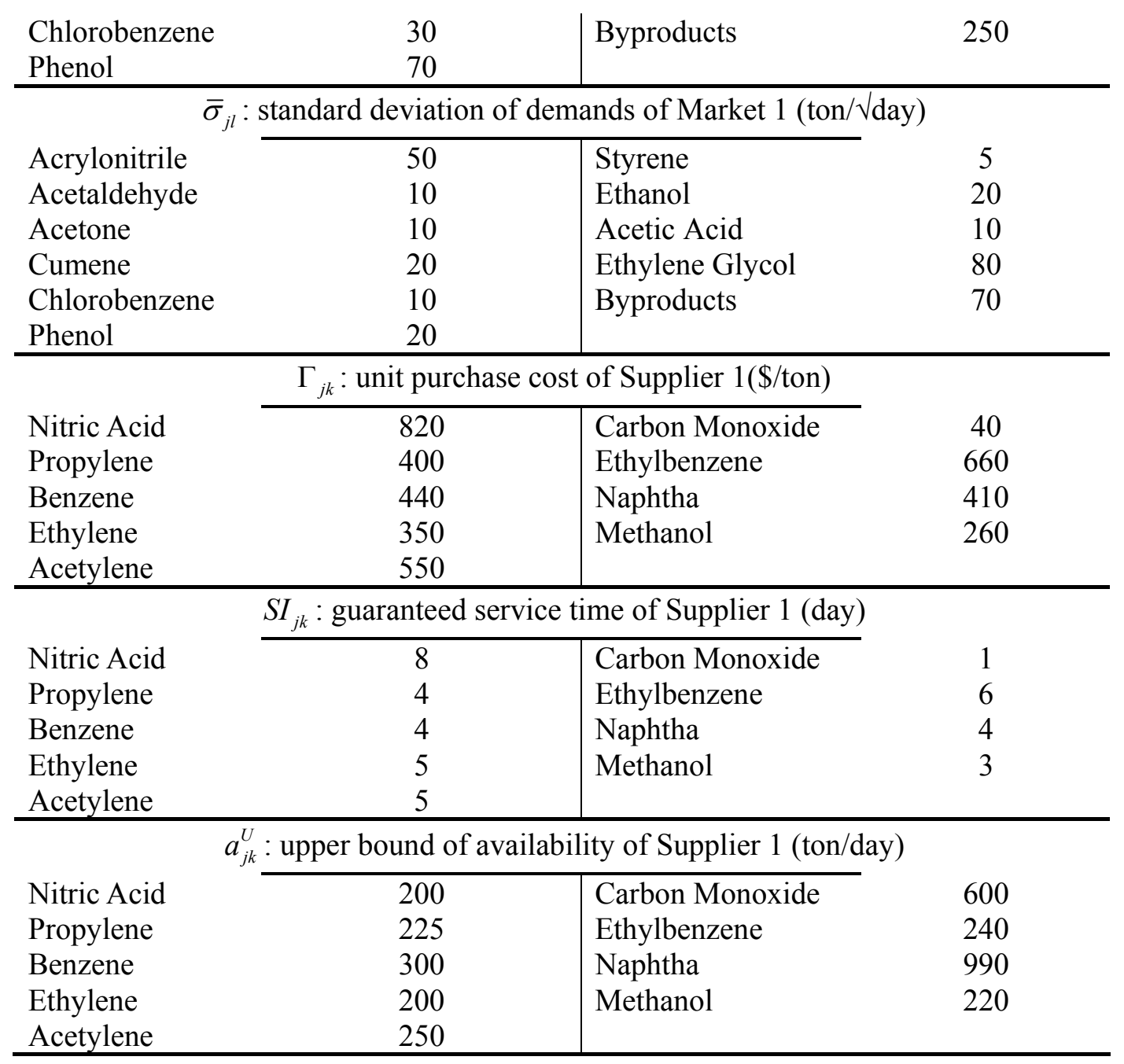

The safety stock factors in all the instances are set to 2.0537 , corresponding to $98 \%$ service level. All the external demands of chemicals in the market follow a normal distribution. The purchase lower bounds, the maximum GSTs of chemicals to the market, and the deterministic transfer times from process $i$ to storage tank of chemical $j\left(\gamma_{i j}\right)$ and from storage tank of chemical $j$ to process $i\left(\theta_{i j}\right)$ are all set to zero. The remaining input data for the first instance with one supplier and one market are given in Table 10. Input data for other instances are available upon request. 
Table 11 Problem sizes for the 10 instances of Example 4

\begin{tabular}{|c|c|c|c|c|c|c|c|c|c|c|}
\hline $\begin{array}{l}\text { Number } \\
\text { of } \\
\text { Suppliers }\end{array}$ & $\begin{array}{c}\text { Number } \\
\text { of } \\
\text { Markets }\end{array}$ & \multicolumn{3}{|c|}{ MINLP Model (P2) } & \multicolumn{3}{|c|}{$\begin{array}{l}\text { MILP Model (P4) for Piecewise linear } \\
\text { Approximation (largest model size) }\end{array}$} & \multicolumn{2}{|c|}{$\begin{array}{r}\text { Reduced (P3) - NLP } \\
\text { (largest model size) }\end{array}$} & $\begin{array}{c}\text { Maximum } \\
\text { Iterations }\end{array}$ \\
\hline 1 & 1 & 9 & 212 & 238 & 179 & 746 & 1,105 & 684 & 962 & 4 \\
\hline 3 & 3 & 27 & 296 & 358 & 190 & 824 & 1,188 & 746 & 1,060 & 4 \\
\hline 4 & 4 & 36 & 338 & 418 & 213 & 880 & 1,266 & 778 & 1,110 & 6 \\
\hline 5 & 5 & 45 & 380 & 478 & 231 & 930 & 1332 & 808 & 1,158 & 5 \\
\hline 7 & 7 & 63 & 464 & 598 & 246 & 1,012 & 1,424 & 870 & 1,256 & 5 \\
\hline 8 & 8 & 72 & 506 & 658 & 259 & 1,058 & 1,482 & 902 & 1,306 & 5 \\
\hline 9 & 9 & 81 & 548 & 718 & 262 & 1,094 & 1,518 & 932 & 1,354 & 4 \\
\hline 10 & 10 & 90 & 590 & 778 & 262 & 1,126 & 1,550 & 964 & 1,404 & 6 \\
\hline
\end{tabular}

"Dis. Var." = discrete variables; "Con. Var." = continues variable; "Const." = constraints. 
Table 12 Comparison of the performance of the algorithms for the 10 instances of Example 4

\begin{tabular}{|c|c|c|c|c|c|c|c|c|c|c|c|}
\hline $\begin{array}{c}\text { Number } \\
\text { of } \\
\text { Suppliers }\end{array}$ & $\begin{array}{c}\text { Number } \\
\text { of } \\
\text { Markets }\end{array}$ & \multicolumn{3}{|c|}{$\begin{array}{c}\text { Solving MINLP Model (P2) with } \\
\text { DICOPT directly }\end{array}$} & \multicolumn{4}{|c|}{$\begin{array}{c}\text { Solving MINLP Model (P2) with } \\
\text { BARON directly }\end{array}$} & \multicolumn{3}{|c|}{ Proposed Algorithm } \\
\hline 1 & 1 & 537,891 & 0.8 & $0.92 \%$ & 532,919 & 531,089 & $0.34 \%$ & 3,600 & 532,919 & 8.3 & 4 \\
\hline 3 & 3 & 485,200 & 4.5 & $0.06 \%$ & 485,200 & 456,645 & $5.89 \%$ & 3,600 & 484,898 & 7.4 & 4 \\
\hline 4 & 4 & 488,231 & 22.4 & $1.73 \%$ & 482,855 & 459,065 & $4.93 \%$ & 3,600 & 479,770 & 52.4 & 6 \\
\hline 5 & 5 & 496,636 & 14.8 & $2.11 \%$ & 511,998 & 458,780 & $10.4 \%$ & 3,600 & 486,146 & 11.3 & 5 \\
\hline 7 & 7 & 496,736 & 20.1 & $6.19 \%$ & 496,317 & 455,754 & $8.17 \%$ & 3,600 & 465,995 & 37.3 & 5 \\
\hline 8 & 8 & $481,114^{*}$ & 42.9 & $0 \%$ & 504,710 & 467,810 & $7.31 \%$ & 3,600 & 481,114 & 52.0 & 5 \\
\hline 9 & 9 & 495,382 & 28.1 & $4.15 \%$ & 497,375 & 457,441 & $8.03 \%$ & 3,600 & 474,814 & 38.4 & 4 \\
\hline 10 & 10 & 524,721 & 67.4 & $10.7 \%$ & 583,188 & 458,796 & $21.3 \%$ & 3,600 & 474,009 & 46.7 & 6 \\
\hline
\end{tabular}

*: infeasible before adding a sufficient small number $\varepsilon$ (e.g. 0.0001) under each square root terms in the objective function 
The problem sizes of all the 10 instances are listed in Table 11. For each instance, we first solve the original non-convex MINLP model (P2) with MINLP solvers BARON and DICOPT directly, and then implement the proposed algorithm by iteratively solving the piecewise linear approximation MILP model (P4) with CPLEX and the reduced model (P3), which is an NLP after fixing the binary variables, with KNITRO. Note that for the MILP model (P4) and the reduced NLP model (P3) after fixing the binary variables, we only report the maximum problem sizes, i.e. the model sizes in the last iteration of the branch-and-refine algorithm. From Table 11, we can see that problem sizes of (P2) increases as the number of suppliers and markets increases. The maximum problems sizes of (P4) and (P3) also increase as the number of markets and suppliers increases, although the number of required iterations for the branch-and-refine algorithm does not strictly increases as the problems sizes increases.

The computational performance of DICOPT, BARON and the proposed algorithm for these 10 instances are presented in Table 12. Note that there are two types of "Gap" in this table. The column "Gap" for solving (P2) with DICOPT is for the differences between the solutions obtained by using DICOPT and the global optimal solutions, which are obtained with the proposed algorithm. The "Gap" column for solving (P2) with BARON is for the differences between the lower and upper bounds returned by BARON.

As we can see from Table 12, DICOPT can solve all the instances in less than 70 seconds, but usually leads to suboptimal solutions with relatively large global optimality margin (up to 10.7\%) for large scale instances. For the second and the eighth instances, DICOPT was not able to return a feasible solution initially due to the numerical difficulty in its NLP subproblems. After adding a sufficiently small number $\varepsilon$ (e.g. 0.0001) under all the square root terms in the objective function, we can avoid unbounded gradient in the NLP subproblems and obtain near-optimal solutions as shown in Table 12. Compared to DICOPT, the performance of the global optimizer BARON is not very good - BARON could not globally optimize any of the 10 instances after running for one hour, and most of the suboptimal solutions returned after 3,600 CPUs have relatively large optimality gaps (in average around $10 \%$ and the maximum is $21.3 \%$ ), although BARON does return feasible solutions for all the instances. Compared with solving (P2) with DICOPT and BARON directly, the proposed algorithm has a significantly better performance. Although the number of iterations may vary from instances to instances (usually $4-6$ iterations), the total CPU 
times for iteratively solving the MILP problem (P4) and the reduced NLP problem (P3) with binary variables fixed, are always less than one minute for all the 10 instances. In addition, the proposed algorithm can guarantee global optimality with similar computational times required by DICOPT. The computational results suggest the significant advantage of using the proposed branch-and-refine algorithm for solving medium and large scale instances. The change of bounds during the branch-and-refine algorithm for the largest instance with 10 suppliers and 10 markets are given in Figure 16. We can see that as iterations proceed, the lower bound keeps increasing, and the upper bound, which is the objective value of a feasible solution, keeps decreasing, until the termination criterion is satisfied. In particular, we can see that the upper bound obtained in the first iteration is rather close to the global optimal solution. Recall that we use the optimal solution of the MILP (P4) as the initial point for solving the NLP (P3) to obtain the upper bounds. The tight upper bound in the first iteration implies that replacing the square root terms in (P3) with the one-piece under-estimator, i.e. the secant, and then solving the resulting MILP problem can lead to very good initial point for solving the NLP, and potentially yield very tight upper bound of the original problem. Similar performance of this approach was also observed and discussed in You and Grossmann. ${ }^{35}$

The optimal solutions of the first instance of Example 4 with 20 chemicals, 13 processes, 1 supplier and 1 market are given in Figure 17 and 18. Figure 17 shows the optimal production level in terms of the production amount of main product, and the unused production capacity of all the processes. We can see that Processes 1, 3, 4, 32 and 38 are operating with almost full capacity, Processes 5, 8, 13, 16, 17 and 28 are using around half of the capacity, while Processes 12 and 14 do not produce any product. The reason is that ethylbenzene is mainly purchased from the supplier instead of being produced from Process 12 due to the price advantage, and acetone is produced from Process 5 instead of Process 14 for lower production cost. Figure 18 shows the optimal inventory levels of all the 20 chemicals in the chemical process network. We can see that intermediates benzene and acetylene, and feedstocks carbon monoxide and methanol have zero inventories, although their downstream chemicals hold sufficiently large safety stocks and cycle stocks. The results show that optimal inventory allocation is a very non-trivial problem for large scale chemical complexes, and it is difficult to use a "rule of thumb" to make such decisions. 
Example 5: Computational Performance, Responsiveness and Pareto Optimization

In the last example, let us consider the large-scale chemical process network discussed at the beginning of this paper and shown in Figure 3. A list of all chemicals included in the chemical complex is given in Table 13 . We still consider $98 \%$ service level for all the chemicals and the maximum GST to the external market are all set to zero. Due to the large size of the problem, the remaining input data are not listed here, but are all available upon request.

Table 13 List of chemicals in Example 5

\begin{tabular}{ll|cl}
\hline A & Nitric Acid & O & Phenol \\
B & Propylene & P & Styrene \\
C & Benzene & Q & Ethanol \\
D & Ethylene & R & Acetic Acid \\
E & Acetylene & S & Ethylene Glycol \\
F & Carbon Monoxide & T & Byproducts \\
G & Ethylbenzene & $\mathrm{U}$ & Vinyl Acetate \\
H & Naphtha & $\mathrm{V}$ & Acetic Anhydride \\
I & Methanol & X & Ethylene Dichloride \\
J & Acrylonitrile & Ethylene Glycol \\
K & Acetaldehyde & Z & Formaldehyde \\
L & Acetone & AA & Ketene \\
M & Cumene & AB & Ethylene Chlorohydrin \\
N & Chlorobenzene
\end{tabular}

Similarly to the previous example, we consider 5 instances with different numbers of suppliers and markets. All the problem sizes are listed in Table 14 and the computational performance of the solvers and the proposed algorithm are shown in Table 15. Similar to what we observed in the previous example, the problem sizes increases as the number of suppliers and markets increases. For these large-scale instances, DICOPT failed to return any feasible solutions without adding a sufficient small number $\varepsilon$ (e.g. 0.0001) in the square root terms of the objective function, due to numerical difficulty in the NLP subproblems. After the minor modification of the objective function, we can avoid unbounded gradients in the NLP subproblems and obtain near-optimal solutions (with optimality gap up to 5.8\%) as shown in Table 15 . The global optimizer BARON still yields relatively large gaps between the lower and upper bounds after running for 3,600 seconds. However, the proposed algorithm is able to globally optimize all the instances with modest CPU times (less than 1,000 seconds), 
and the number of iterations is still modest (2 - 4 iterations) so that the sizes of (P4) and reduced (P3) are not too large. All these five instances again show that the proposed algorithm is able to efficiently solve to global optimality the joint stochastic inventory management and tactical process planning problem, compared to the commercial MINLP solvers.

In addition to illustrating the performance of the proposed algorithm, we address the "responsiveness" issue of this chemical complexes using an approach similar as the one discussed in You and Grossmann. ${ }^{37}$ Since the maximum GST of chemicals to external markets $\left(S O_{j l}^{U}\right)$ is the measure of responsiveness, changing the values of this parameter will lead to changes of the optimal inventory levels and probably the optimal production, purchase and sale levels as well. Thus, we generate 51 instances of this example (with the one supplier and one market instance) by fixing the parameter $S O_{j l}^{U}$ to 51 values evenly distributed in $[0,50]$, i.e. $0,1,2, \ldots \ldots, 49,50$. All the instances are then solved with the proposed algorithm. All the 51 instances require a total CPU time of 1,716.5 seconds. The results produce a Pareto optimal curve shown in the line of Figure 19, which reveals the trade-off between the total daily cost and the measure of responsiveness, the maximum GST to the markets. We can see that as the maximum GST to the markets increases from 0 to 50 days, the optimal total daily cost decreases monotonically from $\$ 397,945 /$ day to $\$ 349,145 /$ day. In particular, there is a steep decrease of the optimal total daily cost from $\$ 397,945 /$ day to $\$ 380,663 /$ day when the maximum GST to the markets increases from 0 to 3 days. Thus, setting the maximum GST to the markets to 3 days might be a good choice in terms of balancing the economics and responsiveness. Similar to the total daily cost, the optimal total inventory also decreases monotonically from 14,391 tons to 0 ton, as the maximum GST to the markets increases from 0 to 50 days. However, the decreasing rate of the optimal inventory level does not strictly follow the one of the total daily cost. It implies that as maximum GST to the markets increases, not only the inventory levels but also the production, purchase and sale activities change in order to reduce the total daily cost. Figure 20a-20f show the optimal safety stocks and cycle stocks for all the chemicals under six different specifications of the maximum GST to the markets ( 0 day, 10 days, 20 days, 30 days, 40 days and 50 days). There is a clear trend that as the maximum GST to the market increases, the inventories of all the chemicals decrease. In particular, we can see in Figure 20a that when the maximum GST to the markets is zero, 
it is optimal to hold sufficiently high inventories for the propylene, benzene and ethylene, which are the major feedstocks of this chemical process network. In addition, there are some inventories for other feedstocks and intermediates, but very few stocks for the final products. Particularly, the optimal inventory levels of acetylene, carbon monoxide, ketene and ethylene chlorohydrin are zero in this case. When the maximum GST to the markets increases to 10 days, the optimal inventory levels for most chemicals decreases to zero, although there are still significant inventories for propylene, benzene and ethylene, and a few stocks for nitric acid, ethylbenzene, naphtha, methanol, acrylonitrile, acetaldehyde and vinyl acetate. If the maximum GST to the markets further increases to 20 days, the optimal inventory level of methanol decreases to zero. When the maximum GST to the markets equals to 40 days, we only need to hold inventory for nitric acid, propylene and acetaldehyde. The optimal inventory levels of all the chemicals are zero when the maximum GST to the markets is 50 days.

\section{Conclusion}

In this paper, we have developed an MINLP model to simultaneously optimize the inventory management decisions and the mid-term process planning decisions for the production, purchase and sale levels with the presence of supply and demand uncertainty. The guaranteed service approach is used to model the inventory system of a chemical complex, and the risk pooling effect is taken into account in the model by relating the demands in the downstream nodes to their upstream nodes. Three illustrative examples are presented to demonstrate the applicability of the proposed model. To efficiently solve the resulting MINLP problem for large scale instances, we exploited some model properties and then proposed an efficient branch-and-refine algorithm based on successive piece-wise linear approximation. Computational experiments on large-scale problems show that the proposed algorithm can obtain global optimal solutions very quickly without the need of a global optimizer.

\section{Acknowledgment}

The authors acknowledge the financial support from the National Science Foundation under Grant No. DMI-0556090 and No. OCI-0750826, and Pennsylvania 
Infrastructure Technology Alliance (PITA). Fengqi You is also grateful to Dr. John M. Wassick at The Dow Chemical Company for helpful discussions at the early stage of this work. 
Table 14 Problem sizes for the 5 instances of Example 5

\begin{tabular}{|c|c|c|c|c|c|c|c|c|c|c|}
\hline $\begin{array}{c}\text { Number } \\
\text { of } \\
\text { Suppliers }\end{array}$ & $\begin{array}{c}\text { Number } \\
\text { of } \\
\text { Markets }\end{array}$ & \multicolumn{3}{|c|}{ MINLP Model (P2) } & \multicolumn{3}{|c|}{$\begin{array}{l}\text { MILP Model (P4) for Piecewise linear } \\
\text { Approximation (largest model size) }\end{array}$} & \multicolumn{2}{|c|}{$\begin{array}{l}\text { Reduced (P3) - NLP } \\
\text { (largest model size) }\end{array}$} & $\begin{array}{l}\text { Maximum } \\
\text { Iterations }\end{array}$ \\
\hline 1 & 1 & 10 & 470 & 522 & 405 & 1,864 & 2,708 & 1,788 & 2,534 & 3 \\
\hline 10 & 10 & 100 & 992 & 1,224 & 494 & 2,384 & 3,264 & 2,166 & 3,092 & 3 \\
\hline 15 & 15 & 150 & 1,282 & 1,614 & 538 & 2,668 & 3,562 & 2,376 & 3,402 & 2 \\
\hline 20 & 20 & 200 & 1,572 & 2,004 & 588 & 2,958 & 3,872 & 2,586 & 3,712 & 2 \\
\hline
\end{tabular}

"Dis. Var." = discrete variables; "Con. Var." = continues variable; "Const." = constraints.

Table 15 Comparison of the performance of the algorithms for the 5 instances of Example 5

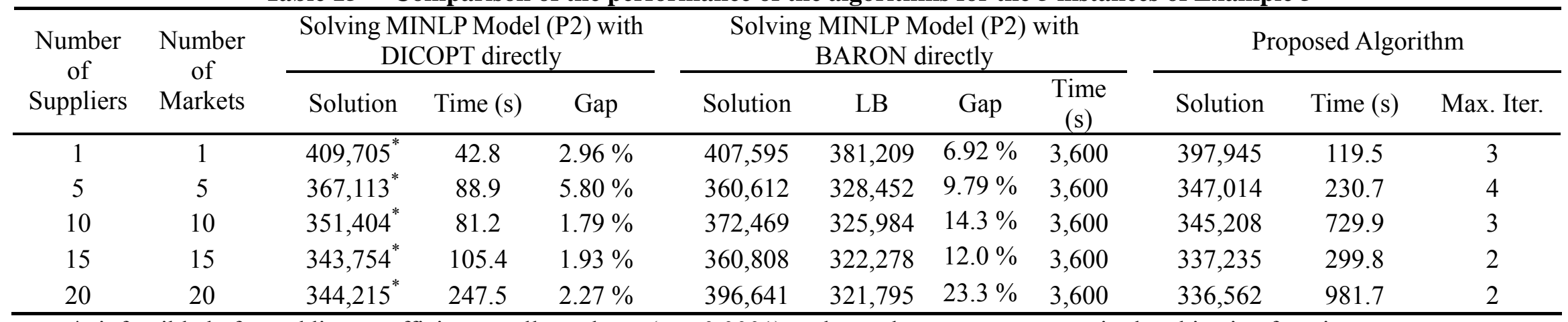

*: infeasible before adding a sufficient small number $\varepsilon$ (e.g. 0.0001) under each square root terms in the objective function 


\section{Appendix: Nomenclature}

\section{Sets/Indices}

I: Set of processes indexed by $i$

$J$ : Set of chemicals indexed by $j$

$K$ : Set of external suppliers indexed by $k$

$L$ : Set of external markets indexed by $l$

$M$ : Set of integers for binary representation indexed by $m$

$P$ : Set of pieces for piece-wise linear approximation indexed by $\mathrm{p}$

\section{Subsets}

$i \in I(j): \quad$ The subset of processes that consume chemical $j$ (for $\eta_{i j}>0$ )

$i \in O(j): \quad$ The subset of processes that produce chemical $j$ (for $\eta_{i j}<0$ )

$j \in C_{i}$ : The subset of chemicals that are input of output of processes $i$

$j \in M_{i}: \quad$ The subset of main products of processes $i$

$k \in S U P(j):$ The subset of external supplier $k$ that supplies chemical $j$

$l \in M K T(j)$ : the subset of external market $l$ that has demand of chemical $j$

\section{Parameters}

$\lambda_{j}: \quad$ Safety stock factor for chemical $j$

$h_{j}$ : Unit inventory holding cost of chemical $j$

$\mathrm{Cap}_{i}$ : Total capacity of process $i$

$\delta_{i}$ : $\quad$ Unit operating cost for process $i$

$P D_{i}$ : $\quad$ Production time delay of process $i$

$\eta_{i j}$ : Mass balance coefficient of chemical $j$ for process $i$

$\bar{\mu}_{j l}: \quad$ Mean value of external demand of chemical $j$ from the market $l$

$\bar{\sigma}_{j l}: \quad$ Standard deviation of external demand of chemical $j$ to the market

$\bar{V}_{j l}: \quad$ Variance of external demand of chemical $j$ from the market $l$

$\bar{R}_{j l}: \quad$ Variance to mean ratio for the demand of chemical $j$ from market $l$ $\left(\bar{R}_{j l}=\bar{\sigma}_{j l}^{2} / \bar{\mu}_{j l}=\bar{V}_{j l} / \bar{\mu}_{j l}\right)$ 
$a_{j k}^{L}$ : Lower bound for the availability of chemical $j$ in the external supplier $k$

$a_{j k}^{U}$ : $\quad$ Upper bound for the availability of chemical $j$ in the external supplier $k$

$\Gamma_{j k}: \quad$ Price of purchase of chemical $j$ from the supplier $k$

$S I_{j k}: \quad$ Guaranteed service time for chemical $j$ of external supplier $k$

$S O_{j l}^{U}: \quad$ Maximum service time for chemical $j$ to external market $l$

$\gamma_{i j}$ : Deterministic transfer time from process $i$ to storage tank of chemical $j$

$\theta_{i j}$ : Deterministic transfer time from storage tank of chemical $j$ to process $i$

$M:$ The maximum positive value such that $P D_{i} / M, S I_{j k} / M, S O_{j l}^{U} / M, \gamma_{i j} / M$, $\theta_{i j} / M$ are all integers

$\alpha_{j p}$ : Piece-wise linear approximation parameter, $\alpha_{j, p}=\frac{\sqrt{u_{j, p}}-\sqrt{u_{j, p-1}}}{u_{j, p}-u_{j, p-1}}$

$\beta_{j p}: \quad$ Piece-wise linear approximation parameter, $\beta_{j p}=\sqrt{u_{j p}}-\alpha_{j p} u_{j p}$

$u_{j p}$ : Parameters for the bounds of intervals in piecewise linear approximation

\section{Binary Variables (0-1)}

$X_{j k}$ : $\quad 0-1$ variable. Equal to 1 if there is positive flow rate from external supplier $k$ to the storage tank of chemical $j$

$Y_{i j l}: \quad 0-1$ variable. Equal to 1 if the variance to mean ratio of raw materials of process $i$ equal to the one of chemical $j$ from market $l$

$Z_{i j m}: \quad 0-1$ variable for the binary representation of the net lead time of chemical $j$ for the demand from downstream process $i$

$E_{j, p}: \quad$ Auxiliary 0-1 variable for piece-wise linear approximation.

\section{Continuous Variables (0 to $+\infty$ )}

$S P_{i j}$ : Guaranteed service time of process $i$ to its downstream storage tank of chemical $j$

$S C_{i j}: \quad$ Guaranteed service time of chemical $j$ to its downstream process $i$

$T C_{j}: \quad$ Worst case replenishment lead time of the storage tank for chemical $j$ 
$T P_{i}: \quad$ Worst case replenishment lead time of process $i$

$S O_{j l}: \quad$ Guaranteed service time of chemical $j$ to external market $l$

$N_{i j}$ : Net lead time of chemical $j$ for the demand from downstream process $i$

$\bar{N}_{j l}: \quad$ Net lead time of chemical $j$ for the demand from external market $l$

$V_{i j}: \quad$ Variance of internal demand of chemical $j$ in process $i$

$W_{i j}$ : Amount of flow of chemical $j$ to/from process $i$

$P u_{j k}: \quad$ Amount of chemical $j$ purchased from external supplier $k$

$S a_{j l}$ : Amount of chemical $j$ sold to external market $l$

$R C_{j}$ : The variance to mean ratio for all the demands of chemical $j$

$R P_{i}$ : $\quad$ Variance to mean ratio for the demands of input chemicals of process $i$

\section{Auxiliary Variables (0 to $+\infty$ )}

$W Y_{i, j, l}: \quad$ Auxiliary variable, $W Y_{i, j, l}=W_{i j} \cdot Y_{i, j, l}$

$W Y 1_{i, j, l}: \quad$ Auxiliary variable

$G_{j}: \quad$ Auxiliary variable, $G_{j}=\sum_{l \in M K T(j)} \bar{N}_{j l} \cdot \bar{V}_{j l}+\sum_{i \in I(j)} N_{i j} \cdot V_{i j}$.

$Q_{j}: \quad$ Auxiliary variable, $Q_{j}=\sum_{l \in M K T(j)} \bar{N}_{j l} \cdot \bar{W}_{j l}+\sum_{i \in I(j)} N_{i j} \cdot W_{i j}$

$Z V_{i j m}: \quad$ Auxiliary variable, for the product of $Z_{i j m}$ and $V_{i j}$.

$Z V 1_{i j m}: \quad$ Auxiliary variable.

$F_{j p}: \quad$ Auxiliary variable for piece-wise linear approximation

\section{References}

1. Wassick JM. Enterprise-wide optimization in an integrated chemical complex. Computers \& Chemical Engineering. 2009; in press.

2. Norton LC, Grossmann IE. Strategic Planning Model for Complete Process Flexibility. Industrial \& Engineering Chemistry Research. 1994; 33:69-76.

3. Sahinidis NV, Grossmann IE. Optimization model for the long range planning in the chemical industry. Computers \& Chemical Engineering. 1989; 13:1049-1063.

4. Ierapetritou MG, Pistikopoulos EN, Floudas CA. Operational planning under uncertainty Computers \& Chemical Engineering. 1996; 20:1499-1516 
5. Jung JY, Blau G, Pekny JF, Reklaitis GV, Eversdyk D. Integrated safety stock management for multi-stage supply chains under production capacity constraints. Computers \& Chemical Engineering. 2008; 32:2570-2581.

6. Liu ML, Sahinidis NV. Optimization in process planning under uncertainty. Industrial \& Engineering Chemistry Research. 1995; 35:4154-4165.

7. Barbaro A, Bagajewicz MJ. Managing Financial Risk in Planning under Uncertainty. AIChE Journal. 2004; 50:963-989.

8. Cheng L, Subrahmanian E, Westerberg AW. Design and planning under uncertainty: issues on problem formulation and solution Computers \& Chemical Engineering. 2003; 27:781-801.

9. Pistikopoulos EN. Uncertainty in process design and operations Computers \& Chemical Engineering. 1995; 19:553-563

10. You F, Grossmann IE. Design of Responsive Supply Chains under Demand Uncertainty. Computers \& Chemical Engineering. 2008; 32:2839-3274.

11. Zipkin PH. Foundations of Inventory Management. McGraw-Hill: Boston, MA, 2000.

12. Grossmann IE. Enterprise-wide Optimization: A New Frontier in Process Systems Engineering. AIChE Journal. 2005; 51:1846-1857.

13. Varma VA, Reklaitis GV, Blau GE, Pekny JF. Enterprise-wide modeling \& optimization - An overview of emerging research challenges and opportunities. Computers \& Chemical Engineering. 2007; 31:692-711.

14. Graves SC, Willems SP. Optimizing strategic safety stock placement in supply chains. Manufacturing \& Service Operations Management. 2000; 2:68-83.

15. Graves SC, Willems SP. Supply chain design: safety stock placement and supply chain configuration. In Handbooks in Operations Research and Management Science, de Kok AG, Graves SC, Eds. Elsevier: North-Holland, Amsterdam, 2003; Vol. 11, pp 95-132.

16. Graves SC, Willems SP. Optimizing the supply chain configuration for new products. Management Science. 2005; 51:1165-1180.

17. Inderfurth K. Safety Stock Optimization in Multi-Stage Inventory Systems. International Journal of Production Economics. 1991; 24:103-113.

18. Eppen G. Effects of centralization on expected costs in a multi-echelon newsboy problem. Management Science. 1979; 25:498-501.

19. You F, P. M. Castro, Grossmann IE. Dinkelbach's algorithm as an efficient method to solve a class of MINLP models for large-scale cyclic scheduling problems. Computers \& Chemical Engineering. 2009; 33:1879-1889.

20. Jackson JR, Grossmann IE. Temporal decomposition scheme for nonlinear multisite production planning and distribution models. Industrial \& Engineering Chemistry Research. 2003; 42:3045-3055.

21. Verderame PM, Floudas CA. Integrated Operational Planning and Medium-Term Scheduling of a Large-Scale Industrial Batch Plants. Industrial and Engineering Chemistry Research. 2008; 47:4845-4860.

22. Ierapetritou MG, Acevedo J, Pistikopoulos EN. An optimization approach for process engineering problems under uncertainty Computers \& Chemical Engineering. 1996; 20:703-709 
23. Bok J-K, Grossmann IE, Park S. Supply Chain Optimization in Continuous Flexible Process Networks. Industrial \& Engineering Chemistry Research. 2000; 39:1279-1290.

24. Chen C-L, Lee W-C. Multi-objective optimization of multi-echelon supply chain networks with uncertain product demands and prices. Computers \& Chemical Engineering. 2004; 28:1131-1144.

25. Gupta A, Maranas CD, McDonald CM. Mid-term supply chain planning under demand uncertainty: customer demand satisfaction and inventory management. Computers \& Chemical Engineering. 2000; 24:2613-2621.

26. Ierapetritoua MG, Pistikopoulosa EN, Floudas CA. Operational planning under uncertainty Computers \& Chemical Engineering. 1996; 20:1499-1516

27. Neiro SMS, Pinto JM. A general modeling framework for the operational planning of petroleum supply chains. Computers \& Chemical Engineering. 2004; 28:871-896.

28. You F, Grossmann IE. Optimal Design and Operational Planning of Responsive Process Supply Chains. In Process System Engineering: Volume 3: Supply Chain Optimization, Papageorgiou LG, Georgiadis MC, Eds. Wiley-VCH: Weinheim, 2007; pp 107-134.

29. You F, Wassick JM, Grossmann IE. Risk Management for Global Supply Chain Planning under Uncertainty: Models and Algorithms. AIChE Journal. 2009; 55:931-946.

30. Simpson KF. In-process inventories. Operations Research. 1958; 6:863-873.

31. Clark A, Scarf S. Optimal policies for a multi-echelon inventory problem. Management Science. $1960 ; 6: 475-490$.

32. Humair S, Willems SP. Optimizing strategic safety stock placement in supply chains with clusters of commonality. Operations Research. 2006; 54:725-742.

33. Magnanti TL, Shen Z-JM, Shu J, Simchi-Levi D, Teo C-P. Inventory placement in acyclic supply chain networks. Operations Research Letters. 2006; 34:228-238.

34. Shu J, Karimi IA. Efficient heuristics for inventory placement in a cyclic networks. Computers \&OperationsResearch. 2009; 36:2899--2904.

35. You F, Grossmann IE. Mixed-Integer Nonlinear Programming Models and Algorithms for Large-Scale Supply Chain Design with Stochastic Inventory Management. Industrial \& Engineering Chemistry Research. 2008; 47: 7802-7817.

36. You F, Grossmann IE. Integrated Multi-Echelon Supply Chain Design with Inventories under Uncertainty: MINLP Models, Computational Strategies. AIChE Journal. 2008; In press.

37. You F, Grossmann IE. Balancing Responsiveness and Economics in the Design of Process Supply Chains with Multi-Echelon Stochastic Inventory. 2009; Submitted.

38. Graves SC. Safety Stocks in Manufacturing Systems. Journal of Manufacturing and Operations Management. 1988; 1:67-101.

39. Inderfurth K, Minner S. Safety stocks in multi-stage inventory systems under different service measures. European Journal of Operations Research. 1998; 106:57-73.

40. Kondili E, Pantelides CC, Sargent RWH. General algorithm for short-term scheduling of batch operations- I. Milp formulation. Computers \& Chemical Engineering. 1993; 17:211-227.

41. Floudas CA, Aggarwal A. A Decomposition Strategy for Global Optimum Search in the Pooling Problem. ORSA JOURNAL ON COMPUTING. 1990; 2:225-235. 
42. Lee HL, Padmanabhan V, Whang; S. The bullwhip effect in supply chains. Sloan Management Review. 1997; 38:93-102.

43. Sahinidis NV. BARON: A general purpose global optimization software package. Journal of Global Optimization. 1996; 8:201-205.

44. Brooke A, Kendrick D, Meeraus A, Raman R, GAMS- A User's Manual. In GAMS Development Corp.: Washington, DC, 1998.

45. Iyer RR, Grossmann IE. A Bilevel Decomposition Algorithm for Long-Range Planning of Process Networks. Industrial \& Engineering Chemistry Research. 1998; 37:474-481.

46. Minner S. Strategic safety stocks in reverse logistics supply chains. International Journal of Production Economics. 2001; 71:417-428.

47. Falk JE, Soland RM. An Algorithm for separable nonconvex programming problems. Management Science. $1969 ; 15: 550-569$.

48. Schectman JP, Sahinidis NV. A Finite Algorithm for Global Minimization of Separable Concave Programs. Journal of Global Optimization. 1998; 12:1-36.

49. Nemhauser GL, Wolsey LA. Integer and Combinatorial Optimization. John Wiley and Son Inc.: New York, NY, 1988.

50. Glover F. Improved Linear Integer Programming Formulations of Nonlinear Integer Problems. Management Science. 1975; 22:455-460.

51. Bergamini ML, Grossmann IE, Scenna N, Aguirre P. An improved piecewise outer-approximation algorithm for the global optimization of MINLP models involving concave and bilinear terms. Computers \& Chemical Engineering. 2008; 32:477-493.

52. Croxton KL, Gendron B, Magnanti TL. A Comparison of Mixed-Integer Programming Models for Nonconvex Piecewise Linear Cost Minimization Problems. Management Science. 2003; 49:1268-1273.

53. Padberg MW. Approximating separable nonlinear functions via mixed zero-one programs. Operations Research Letters. 2000; 27:1-5.

54. Wicaksono DS, Karimi IA. Piecewise MILP under- and overestimators for global optimization of bilinear programs. AIChE Journal. 2008; 54:991 - 1008. 